\title{
Review Article \\ The Distorted-Wave Born Approach for Calculating Electron-Impact Ionization of Molecules
}

\author{
Don H. Madison and Ola Al-Hagan \\ Department of Physics, Missouri University of Science and Technology, Rolla, MO 65409, USA \\ Correspondence should be addressed to Don H. Madison, madison@mst.edu
}

Received 29 November 2009; Accepted 4 January 2010

Academic Editor: Derrick S. F. Crothers

Copyright ( $) 2010$ D. H. Madison and O. Al-Hagan. This is an open access article distributed under the Creative Commons Attribution License, which permits unrestricted use, distribution, and reproduction in any medium, provided the original work is properly cited.

The distorted-wave Born approximation (DWBA) has been one of the most successful theoretical approaches for treating electron collisions with complicated atoms, and recently the DWBA has been successfully extended to treat electron-impact ionization of molecules. The purpose of this paper is to give an overview of that development and to provide a summary of the recent experimental and theoretical works examining low to intermediate energy electron-impact single ionization of molecules.

\section{Introduction}

One of the most fundamental, unsolved problems in physics is the few-body problem. The few-body problem arises from the fact that the Schrödinger equation is not analytically solvable for more than two mutually interacting particles. As a result, for three or more particles, theory must resort to significant modeling efforts using approximations, the validity of which is determined by comparison with experiment.

Atomic collisions present a valuable test of these theoretical treatments of the few-body problem for two main reasons. First, the underlying fundamental interaction between the particles in atomic systems, the electromagnetic force, is well understood. In particular, for any two particles, it is known exactly. Consequently, for collisions involving more than two particles, discrepancies between experiment and theory must be attributed to the fewbody aspects of the theoretical model. Second, the recent advancements in experimental techniques, such as coldtarget recoil-ion momentum spectroscopy (COLTRIMS) [1-5] have allowed for complete kinematic information about every particle in the system to be determined. These fully differential cross-section (FDCS) measurements provide a stringent test for even the best theoretical models.
Advances on the theoretical side now allow for an essentially exact numerical calculation of one of the simplest three-body problems, namely, electron-impact ionization of hydrogen [6-8]. Single ionization of helium with the ion being left in the ground state can also be treated as a 3-body problem, and very good agreement between experiment and theory has been achieved for electron-impact ionization of helium as well [9-12]. However, for heavy particle ionization of helium, agreement between experiment and theory is not as good, particularly for out-of-plane scattering [5]. For single ionization of heavier atoms, and particularly molecules, approximations have to be made. This has led to the development of a number of theoretical models that deal with different targets, impact energies, and geometries. Each model uses different approximations and as a result, the experiments play an important role in verifying the accuracy of theoretical approximations and guiding the calculations.

One of the most successful theoretical approaches for electron-impact ionization of more complicated targets is the distorted-wave Born approximation (DWBA). The DWBA treats single ionization of a complex target as a 3-body problem with the effect of the spectator electrons being represented by a spherically symmetric potential which is used in the Schrödinger equation to calculate the continuum wave functions for the continuum particles. Madison et al. [13] reported the very first DWBA calculation for ionization 
of helium in 1977 and numerous DWBA papers can be found in the literature (for a representative sample of DWBA papers since 1990 see [14-100]). In general, the DWBA tends to give reasonable agreement with the dominant features of the fully differential cross-section (FDCS) measurements for electron-impact ionization of atoms if the incident electron has an energy of about $100 \mathrm{eV}$ or larger.

In the standard DWBA for ionization, the final state wave function is represented as a product of two wave functions which contain no mutual electron-electron repulsion (normally called postcollision interaction or PCI). In 1989, Brauner et al. [101] (to be referred to as BBK) showed that significantly better agreement with experiment could be achieved for electron-impact ionization of atomic hydrogen at lower energies if the PCI was included directly in the final state wave function instead of just the perturbation. Including the PCI in the final state wave function is accomplished by including the Coulomb interaction between the two final state continuum electrons in the final state wave function. The big advantage of including the electronelectron interaction directly in the final state wave function stems from the fact that any physics contained in the wave function is automatically contained to all orders of perturbation theory so the BBK treatment contains the PCI to all orders of perturbation theory.

The BBK final state wave function for ionization of hydrogen is a product of three Coulomb functions-a Coulomb wave for each of the two continuum electrons in the field of a proton and the Coulomb interaction between the two electrons. This 3-Coulomb (3C) wave function was first proposed by Redmond [102] and Rosenberg [103] almost 40 years ago and one of the attractive features of the $3 \mathrm{C}$ wave function lies in the fact that it is an exact asymptotic solution of the 3-body problem. The success of the BBK work inspired investigations of other wave functions for 3continuum particles [104-107]. Alt and Mukhamedzhanov [104] proposed a wave function using local relative momenta which was shown to also be asymptotically correct. Berakdar [105] proposed the dynamic screened 3C (DS3C) wave function. In the DS3C wave function, the momenta are constants while the effective charges are dynamical variables. These charges are chosen such that the wave function has the proper behavior on the Wannier ridge.

The $T$-matrix that needs to be evaluated to calculate the cross-section is a 6-dimensional (6D) integral. However, in the standard DWBA without the PCI, all the angular integrals can be evaluated analytically and only two radial integrals are performed numerically which means that DWBA results can be generated in a few minutes on a single standard processor. On the other hand, if the Coulomb interaction depending on relative electron-electron coordinates is put in the final-state wave function, the angular integrations can no longer be performed analytically which means that a more complicated integral is required. In the BBK work for ionization of atomic hydrogen, all the wave functions were analytic which allowed for a partial analytic integration of the $T$-matrix which simplified the required numerical work.

If the final state Coulomb interaction is included in the standard DWBA approximation using numerical bound and continuum wave functions, a full $6 \mathrm{D}$ integral is required for the $T$-matrix. Due to the numerical difficulties associated with evaluating 6D integrals, several approximations have been proposed to simplify the integral. The Coulomb interaction between the two electrons can be expressed as a product of the Gamow factor and $\mathrm{a}_{1} \mathrm{~F}_{1}$ hypergeometric function and it is the hypergeometric function that causes the problem. Botero and Macek [108] (see also Whelan et al. $[31,32])$ proposed neglecting the hypergeometric function and just using the Gamow factor. With this approximation, the electron-electron repulsion factors out of the integral and the net effect are to multiply the DWBA amplitude by the Gamow factor. Kheifets et al. [95, 96] recently showed that approximating the Coulomb interaction by the Gamow factor significantly improved agreement between experiment and theory for high energy ionization of inert gases particularly at larger scattering angles. Ward and Macek [109] proposed a low energy approximation keeping the hypergeometric function but evaluating it for an average separation between the electrons. Al-Hagan et al. [110] recently found that this was a good approximation for low energy ionization of molecular hydrogen. Jones et al. [29] argued that the full strength Coulomb interaction between the two continuum electrons may be too strong for small separations so they suggested using the asymptotic form for the hypergeometric function. All of these approximations allow one to include PCI while keeping the calculation difficulty at the same level as the DWBA.

All of the above work focused on approximations for the final state wave function. The BBK approximation uses a plane wave for the incoming electron which is assumed to be $\mathrm{OK}$ at least for high energies since it is asymptotically correct. However, for heavy ion impact, Crothers and McCann [111] proposed using an Eikonal initial state (EIS) and this approximation has had huge success for double differential cross-sections (DDCS) for heavy particle ionization. Jones and Madison $[112,113]$ investigated different possible initial states for electron-impact ionization of atomic hydrogen and they found that the EIS with the $3 \mathrm{C}$ final state gave the best agreement with experiment for electron impact ionization as well. In the heavy particle calculations, approximations appropriate for heavy particle DDCS were made such as straight line paths and neglecting some terms in the $T$ matrix. Since these approximations would not be appropriate for FDCS for electron scattering, Jones and Madison performed a full quantum mechanical calculation including all terms in the $T$-matrix.

Subsequently, we successfully applied the same EIS approach to recent 3D FDCS measurements for heavy particle ionization [5, 114-116]. Crothers and McCann labeled their approximation continuum distorted-waveEikonal initial state (CDW-EIS). Our approach differed from the numerous CDW-EIS calculations performed for heavy particle scattering in three ways.

(1) All of the CDW-EIS calculations performed in the 1990s for less differential cross-sections used a straight-line semiclassical trajectory for the projectile while we use a full quantum-mechanical treatment. 
These straight-line semiclassical trajectory models have been very effective in predicting experimental data for less differential cross-sections. However, these approximations have been shown to be not as reliable for cross-sections differential in the projectile scattering angle [117-124].

(2) We use distorted-waves for the continuum states of the active electron. The previous studies have shown that distorted-waves can improve CDW-EIS calculations in less differential cross-sections [125]. Madison et al. [114] showed that distorted-waves are even more important for FDCS studies.

(3) The exact $T$-matrix can be expressed as the sum of two terms, and all the previous CDW-EIS calculations evaluate only the first term of the sum whereas we evaluate both terms.

Consequently, we labeled our calculation 3DW-EIS to distinguish from the previous CDW-EIS calculations.

For electron-impact ionization of heavier atoms or molecules, it is necessary to use numerical bound state wave functions and numerical distorted-waves for the continuum electrons. If one additionally wants to include the final state electron-electron Coulomb interaction in the final state, one is forced to perform a full numerical $6 \mathrm{D}$ integral for the $T$ matrix. Due to the complexity of performing such an integral [126], it was not until 2003 before the first DWBA calculation including PCI was reported by Prideaux and Madison [75] for ionization of argon and krypton. Prideaux and Madison called the DWBA calculation including the full Coulomb PCI the 3-body distorted-wave (3DW) approximation. The difference in computer time between the DWBA and 3DW calculations is a few minutes versus a few days on a single processor.

All the above calculations are based upon first-order perturbation theory. However, one has to be very careful with terminology since any physics contained in the initial or final state wave function is contained to all orders of perturbation theory while the interactions in the perturbation are contained to only first-order. As a result, a first-order calculation will normally contain some physics to all orders. For example, a distorted-wave is an elastic scattering wave function from the target. Consequently, a normal DWBA calculation will contain elastic scattering of the incoming electron from the neutral target to all orders of perturbation theory as well as elastic scattering of the two final continuum electrons. The inclusion of the full Coulomb PCI in the final state wave function means that the final-state electronelectron repulsion is included to all orders of perturbation theory.

Although there were some second-order plane-wave Born calculations (PWB2) using closure reported in the 1980s for ionization of hydrogen [127], most of the more recent second-order work has concentrated on two electron process for electron-helium collisions. Marchalant et al. [128-130] reported a PWB2 calculation for excitationionization of helium in which the first-order term was evaluated highly accurately and closure was used for the secondorder term. Bartschat and coworkers [131-134] reported second-order hybrid distorted-wave $R$-matrix (RM) calculations (DWB2-RM) which use the closure approximation and the simplified Green's (SG) function approximation to evaluate the second-order term. The only DWB2 calculation which has evaluated the second-order term without making any approximations (other than using pseudostates for the intermediate states) was that of Chen et al. [135] and Chen and Madison [136]. Although the second-order term was evaluated more accurately in the Chen DWB2 work, the firstorder term was not as accurate as either of the above PWB2 and DWB2-RM works so it is difficult to make meaningful comparisons. Chen et al. [137] evaluated the validity of the closure and simplified Green's function approximations and, not surprisingly, found that the validity of these approximations strongly depended on the kinematics of the collision.

Electron-impact single ionization of molecules has been extensively studied for 2-3 decades using high-energy incident electrons. This work has been nicely summarized by Weigold and McCarthy [138]. It is now well known that, for high energy, the FDCS is proportional to the square of the momentum space wave function for the active orbital averaged over all orientations (the so-called Dyson orbital) and, as a result, the high energy studies are normally called electron momentum spectroscopy (EMS). These EMS studies have provided a wealth of information about the quality of quantum chemistry calculations of molecular wave functions. For high energy, the dynamics of the collision is not important (i.e., the Dyson orbital is independent of the incident electron energy).

Although there were some experimental papers reported for low to intermediate energy ionization of molecules in the $90 \mathrm{~s}$, atoms received more attention probably due to limited theoretical support for the molecular measurements. The challenge for molecules is to use a multicenter wave function that is normally not spherically symmetric as compared to atoms which have one scattering center and spherically symmetric wave functions. Another challenge arises from the fact that the experiments to date do not align the molecules before the collision. The traditional $(e, 2 e)$ measurements therefore represent an average over all molecular orientations which must be taken into account in the theoretical approach. In spite of these difficulties, there has been an increased interest in low energy molecular ionization in the last ten years [139-181].

On the theoretical side, the plane-wave impulse approximation (PWIA) developed by McCarthy and coworkers $[138,182-184]$ has been very successful in the EMS work for studying molecular structure. Other theoretical approaches include the approach of Robicheaux [185] who introduced an analytical method to treat electron-impact ionization of $\mathrm{H}_{2}{ }^{+}$using a prolate spheroidal coordinate system. This method is a useful way to assess the experimental data and other approximations. The modified additive rule (MAR) obtains molecular ionization cross-sections by summing the ionization cross-section for each constituent atom using atomic weighting factors. MAR was used to obtain theoretical cross-sections for electron-impact ionization of $\mathrm{C}_{2} \mathrm{H}_{6}$ by Deutch and Becker [186]. 
The distorted-wave impulse approximation (DWIA) is similar to the PWIA except that the plane waves are replaced with distorted-waves. Gao et al. presented DWIA results for electron-impact ionization of $\mathrm{N}_{2}$ [156] for incident electron energies between $35.6 \mathrm{eV}$ and $400 \mathrm{eV}$. The DWIA results were compared to the experimental data of Rioual et al. [187] as well as Hussey and Murray [147]. There was reasonable agreement at intermediate to high incident electron energies. However, the agreement worsened at low incident electron energies. Comparing the PWIA and the DWIA, the DWIA gave better agreement with the experimental data particularly at the intermediate energies.

In the first Born approximation (FBA), the ejected electron is treated as a Coulomb wave whereas the incident and scattered electrons are treated as plane waves. In 2001, Champion et al. [142] used the FBA to study electronimpact ionization of $\mathrm{H}_{2} \mathrm{O}$. Monzani et al. [188] reported a DWBA approach for $\mathrm{H}_{2}$ in which all incoming and outgoing continuum electrons are represented as distorted-waves calculated in a single-center static-exchange potential. Weck et al. [141, 150] developed the first Born approximation twocenter continuum (FBA-TCC) approximation with correct boundary conditions in the entrance and exit channels. In the TCC approximation for diatomic molecules, one assumes that the ejected electron is ionized from the proximity of one of the nuclei and the passive electron completely screens the other nucleus. As a result, the ejected electron interacts with only one nucleus and the projectile electron. In this approach the incident and scattered electrons are represented as plane waves. This method was quite successful in reproducing the high energy $(\sim 4 \mathrm{keV}) \mathrm{H}_{2}$ absolute experimental data of Chérid et al. [189]. Most recently Colgan et al. [177179] generalized the time dependent close coupling (TDCC) method previously used for atoms to ionization of molecular hydrogen.

We generalized the 3DW approach we had developed for atoms to molecules which we called the molecular 3body distorted-wave approach (M3DW) [157, 158]. Similar to atoms, the key ingredient for the M3DW is the electronic charge distribution of the target and the orbital for the active electron. However, very different from atoms, the charge distributions and orbitals cannot be expressed in terms of radial functions and spherical harmonics but rather in terms of a numerical 3D grid. Similar to atoms, static potentials for the molecular electrons are obtained by averaging the charge distribution over all possible orientations. For atoms the nucleus is treated as a point charge while for molecules we have to again average over all possible orientations and this average places the charge of the nucleus on a sphere of radius $R$, where $R$ is the distance of the nucleus from the center of mass. The static potential is the sum of the electronic plus nuclear parts and this potential is used to solve the Schrödinger equation for the continuum electrons (similar to atoms). Since the charge distributions and molecular orbitals depend on the orientation of the molecule, the fundamental FDCS depends on the orientation of the molecule. Although there is presently considerable experimental effort directed at measuring FDCS which depend on molecular orientation, the traditional $(\mathrm{e}, 2 \mathrm{e})$ measurements represent an average over all molecular orientations. Calculating a proper average over all orientations using the M3DW represents a significant computer challenge since a few days are required to get the results for a single orientation (using a single processor). Consequently, Gao et al. [157] proposed the orientation-averaged molecular orbital (OAMO) approximation in which a single average molecular orbital is used to approximate the average over all orientations. While this approximation is not good for most molecular states, it has proved to be very successful for a few highly symmetric states [110, 156-159, 164-169].

The purpose of this paper is to give an overview of the M3DW method for ionization of molecules and to show the current status of agreement between experiment and theory for low to intermediate energy electron-impact ionization of molecules.

\section{Three-Body Distorted-wave Approximation for Molecules}

The theoretical basis for the 3-body distorted-wave approximation was described by Prideaux and Madison [75] for ionization of atoms. That approach was generalized to molecules by Gao et al. [156, 157]. We start with the exact $T$-matrix for the ionization process which can be written as

$$
T=\left\langle\Psi_{f}\left|H-H_{0}\right| \Phi_{i}\right\rangle
$$

where $H$ is the full Hamiltonian for the system, $H_{0}$ is an approximate initial-state Hamiltonian, and the wave functions $\Psi_{f}$ and $\Phi_{i}$ in the $T$-matrix are eigenfunctions of the two Hamiltonians

$$
\begin{gathered}
H\left|\Psi_{f}\right\rangle=E\left|\Psi_{f}\right\rangle, \\
H_{0}\left|\Phi_{i}\right\rangle=E\left|\Phi_{i}\right\rangle .
\end{gathered}
$$

In terms of the physics contained in the $T$-matrix, any interaction which is included in the calculation of the initialand final-state wave functions is contained to all orders of perturbation theory for that channel while any interactions contained in the operator $\left(H-H_{0}\right)$ (normally called the perturbation) are contained to first-order in perturbation theory. To evaluate the $T$-matrix, one must choose $H_{0}$ and approximate $\Psi_{f}$.

One of the most successful approximations for calculating atomic ionization by electron impact has been the firstorder distorted-wave Born approximation (DWBA). In the standard DWBA, the initial-state Hamiltonian is chosen to be

$$
H_{0}=H_{\text {target }}+T_{p}+U_{i}
$$

where $H_{\text {target }}$ is the Hamiltonian for the neutral target with eigenfunctions $\psi_{\text {target }}, T_{p}$ is the kinetic energy operator for the projectile, and $U_{i}$ is an initial-state spherically symmetric potential for the projectile-target interaction (normally called the initial-state distorting potential). The initial-state distorting potential consists of the nuclear contribution plus 
a spherically symmetric approximation for the interaction between the projectile electron and the target electrons which is obtained from the quantum mechanical charge density of the target. The initial-state distorted-wave $\chi_{i}$ is an eigenfunction obtained from the initial-state distorting potential

$$
\left(T_{p}+U_{i}\right) \chi_{i}=\varepsilon_{i} \chi_{i}
$$

where $\varepsilon_{i}$ is the energy of the incoming projectile. The physics contained in the initial state distorted-wave is elastic scattering of the projectile from the neutral target represented by the effective potential $U_{i}$. In the normal DWBA, the exact final-state wave function is approximated as a product of wave functions for each of the final three particles

$$
\Psi_{f} \approx \chi_{\text {proj }} \chi_{\text {eject }} \psi_{\text {ion }} .
$$

Here $\psi_{\text {ion }}$ is the final-state wave function for the ion, and the final-state distorted-waves $\chi_{\text {proj }}\left(\chi_{\text {eject }}\right)$ are obtained from the final-state distorting potential $U_{\text {ion }}$

$$
\left(T_{p}+U_{\text {ion }}\right) \chi_{\text {proj(eject })}=\varepsilon_{a(b)} \chi_{\text {proj(eject })},
$$

where $\varepsilon_{a(b)}$ is the energy of the scattered (ejected) electron. The final-state distorting potential $U_{\text {ion }}$ consists of the nuclear contribution plus a spherically symmetric approximation for the interaction between the continuum electron and the bound electrons in the ion. The physics contained in the final state distorted-wave is elastic scattering of the continuum electron from the final state ion represented by the effective potential $U_{\text {ion }}$.

The full Hamiltonian is given by

$$
H=H_{\text {target }}+T_{p}+V_{i}
$$

where $V_{i}$ is the initial state interaction between the projectile and target. As a result, the direct-scattering DWBA $T$-matrix for molecules is given by

$$
T_{\text {dir }}^{\mathrm{MDW}}=\left\langle\chi_{\text {proj }} \chi_{\text {eject }} \psi_{\text {ion }}\left|V_{i}-U_{i}\right| \psi_{\text {target }} \chi_{i}\right\rangle .
$$

As mentioned above, any physics contained in the wavefunctions of the $T$-matrix is contained to all orders of perturbation theory. For the initial state, the distortedwave $\chi_{i}$ is an eigenfunction of $U_{i}$ which means that elastic scattering from the nuclei plus elastic scattering from the spherically symmetric effective potential for all the bound electrons is contained to all orders of perturbation theory. Likewise, for the final state, the distorted-waves $\chi_{\text {proj }}\left(\chi_{\text {eject }}\right)$ are eigenfunctions of $U_{\text {ion }}$ which means that elastic scattering from the nuclei plus elastic scattering from the spherically symmetric effective potential for the bound electrons in the ion is contained to all orders of perturbation theory. As will be discussed below, in the 3-body approximation, the physics contained in $\left(V_{i}-U_{i}\right)$ is the nonspherical part of the projectile-active-electron interaction so this is the only physics contained to first-order.
The DWBA has been highly successful for calculating the FDCS for ionization by higher-energy electrons. However, as the energy of the electron decreased, the DWBA starts to fail. One source of this failure is an inadequate treatment of the final-state interaction between the projectile electron and ejected electron. As mentioned in the introduction, BBK demonstrated that better agreement with experiment for electron-hydrogen scattering could be achieved for lower incident electron energies by including the final-state projectile-electron interaction in the approximation for the final-state wave function. In the BBK approach, the exact final state for electron-hydrogen scattering is approximated as

$$
\Psi_{f} \approx \mathrm{CW}_{\text {proj }} \mathrm{CW}_{\text {eject }} \mathrm{C}_{\text {proj-eject }},
$$

where CW is a Coulomb wave for an electron in the field of a proton and $\mathrm{C}$ is the Coulomb distortion factor which contains the effects of the final-state Coulomb interaction between the projectile and the ejected electron (PCI). The wave function (9) is called the 3C wave function. For heavier atoms or molecules, a generalization of the $3 \mathrm{C}$ to the distorted-wave approach is required. The DWBA equivalent of the $3 \mathrm{C}$ wave function for the final-state wave function would be

$$
\Psi_{f} \approx \chi_{\text {proj }} \chi_{\text {eject }} \mathrm{C}_{\text {scat-eject }} \psi_{\text {ion }} .
$$

Asymptotically this wave function would be a phase-shifted 3C wave function. We will call the wave function (10) a 3body distorted-wave (3DW). One of the attractive features of this wave function is that it is an exact asymptotic solution of the three body problem. The direct-scattering molecular 3-body distorted-wave $T$-matrix with the final-state wave function (10) is given by

$$
T_{\text {dir }}^{\mathrm{M} 3 \mathrm{DW}}=\left\langle\chi_{\text {proj }} \chi_{\text {eject }} \mathrm{C}_{\text {scat-eject }} \psi_{\text {ion }}\left|V_{i}-U_{i}\right| \psi_{\text {target }} \chi_{i}\right\rangle .
$$

The physics contained in M3DW approximation is the following. The final-state Coulomb interaction between the projectile and a screened nuclear charge, the Coulomb interaction between the ejected-electron and a screened nuclear charge, and the Coulomb interaction between the projectile and ejected-electron are contained to all orders of perturbation theory. For the initial state, the Coulomb interaction between the projectile and a screened nuclear charge for a neutral target is contained to all orders of perturbation theory. Similar to the DW, the only interaction contained only to first-order in the 3DW is the initial-state nonspherical projectile-active-electron interaction as will be demonstrated below.

In the DW approach, ionization of more complex targets is treated as a three-body problem. In the 3-body approach, the initial-state interaction is approximated as

$$
V_{i}=\frac{1}{r_{a b}}+U_{\text {ion }} .
$$

Here $\left(1 / r_{a b}\right)$ represents the interaction between the projectile electron and the active target electron and $U_{\text {ion }}$ is the 
interaction between the projectile electron and the rest of the target including the nuclei. The initial-state distorting potential is given by

$$
U_{i}=U_{a}+U_{\text {ion }}
$$

where $U_{a}$ is the spherically symmetric interaction potential between the projectile and the active electron. As a result the perturbation is given by

$$
V_{i}-U_{i}=\frac{1}{r_{a b}}-U_{a} .
$$

From (14), it is seen that the perturbation is the difference between the full interaction between the projectileactive electron and the spherically symmetric approximation for this interaction. Hence the perturbation is the nonspherical part of the projectile-active electron interaction as mentioned above. Since $U_{a}$ depends only on the radial distance of the projectile $U_{a}\left(r_{a}\right)$, the perturbation depends only on the coordinates of the projectile and active electron. If we let $\xi$ represent the coordinates of all the passive electrons, the final state ion wave function for the molecule $\psi_{\text {ion }}(\xi, \mathbf{R})$ will depend on $\xi$ and the orientation of the molecule $\mathbf{R}$, while the initial target wave function $\psi_{\text {target }}\left(\xi, \mathbf{r}_{a}, \mathbf{R}\right)$ will depend on both $\xi$ and $\mathbf{R}$ and the active electron $\mathbf{r}_{a}$ (we assume that the collision time is sufficiently short so that the final-state orientation is the same as the initial-state orientation). Consequently the integral over the passive electron coordinates is

$$
\left\langle\psi_{\text {ion }}(\xi, \mathbf{R}) \mid \psi_{\text {target }}\left(\xi, \mathbf{r}_{a}, \mathbf{R}\right)\right\rangle=\psi_{\text {Dyson }}\left(\mathbf{r}_{a}, \mathbf{R}\right),
$$

where $\phi_{\text {Dyson }}\left(\mathbf{r}_{a}, \mathbf{R}\right)$ is the so-called Dyson orbital which depends on the orientation of the molecule R. Consequently, the direct-scattering $T$-matrix of (11) depends on the orientation of the molecule

$$
\begin{aligned}
T_{\text {dir }}^{\mathrm{M} 3 \mathrm{DW}}(\mathbf{R})=\langle & \chi_{\text {proj }}\left(\mathbf{r}_{a}\right) \chi_{\text {eject }}\left(\mathbf{r}_{b}\right) \mathrm{C}_{\text {scat-eject }}\left(\mathbf{r}_{a b}\right) \mid \frac{1}{r_{a b}} \\
& -U_{a}\left(r_{a}\right)\left|\psi_{\text {Dyson }}\left(\mathbf{r}_{a}, \mathbf{R}\right) \chi_{i}\left(\mathbf{r}_{b}\right)\right\rangle .
\end{aligned}
$$

As mentioned in the introduction, evaluating the DW $T$-matrix of (8) takes a few minutes on a single processor while evaluating (16) can take a few days. Almost all of the experimental data reported so far represents an average over all molecular orientations and the proper way to calculate an average over orientations would be to evaluate (16) at a sufficiently large number of orientations that a numerically accurate average could be calculated. Due to the excessive computer time required for this process, Gao et al. [157] proposed the orientation-averaged molecular orbital (OAMO) approximation. The essence of the OAMO approximation is to average the molecular orbitals instead of averaging the cross-sections. In this approximation, the calculation of molecular $(\mathrm{e}, 2 \mathrm{e})$ cross-sections reduces to the same level of difficulty as calculating atomic cross-sections

$$
\begin{gathered}
T_{\mathrm{dir}}^{\mathrm{M} 3 \mathrm{DW}}=\left\langle\chi_{\mathrm{proj}}\left(\mathbf{r}_{a}\right) \chi_{\text {eject }}\left(\mathbf{r}_{b}\right) \mathrm{C}_{\text {scat-eject }}\left(\mathbf{r}_{a b}\right)\right| \frac{1}{r_{a b}} \\
-U_{a}\left(r_{a}\right)\left|\psi_{\text {Dyson }}^{\text {OAMO }}\left(r_{a}\right) \chi_{i}\left(\mathbf{r}_{b}\right)\right\rangle,
\end{gathered}
$$

where $\psi_{\text {Dyson }}^{\mathrm{OAMO}}\left(r_{a}\right)$ is the Dyson orbital averaged over all orientations. While the OAMO approximation is not valid for most molecular orbitals, Gao et al. [157] showed that it is valid for highly symmetric orbitals as long as the momentum transferred to the ion is less than unity (i.e., near the binary peak).

The spherically symmetric distorting potentials for molecules are calculated similar to the atomic case. The starting point is the molecular charge density for the neutral molecule which is obtained from the Dyson orbitals

$$
\rho(\mathbf{r}, \mathbf{R})=\sum_{k=1}^{m} n_{k}\left|\phi_{\text {Dyson }}^{k}(\mathbf{r}, \mathbf{R})\right|^{2},
$$

where $m$ is the number of orbitals in the molecule, $n_{k}$ is the occupation number of the orbital, and the density depends on the orientation of the molecule. We initially calculated the Dyson orbitals using the computer code GAMESS with a small basis set. More recently, we formed a collaboration with C. G. Ning who calculates more accurate wave functions using density function theory with a B3LYP/TZ2P basis set. To obtain the spherically symmetric distorting potential, we average (18) over all orientations to form the average radial charge density

$$
\rho^{\mathrm{ave}}(r)=\langle\rho(\mathbf{r}, \mathbf{R})\rangle,
$$

where the brackets denote taking an average over all orientations. The spherically symmetric static distorting potential representing the interaction between the projectile-electron and the target molecular electrons is then found in the standard way using the average radial density

$$
U_{\text {ele }}\left(r_{a}\right)=\left\langle\int \frac{\rho^{\text {ave }}(r) d \mathbf{r}}{\left|\mathbf{r}_{a}-\mathbf{r}\right|}\right\rangle,
$$

where now the brackets denote taking an average over all angular locations for $\mathbf{r}_{a}$. The initial state static distorting potential is the sum of the electronic contribution plus the nuclear contribution

$$
U_{\text {static }}=U_{\text {ele }}+U_{\text {nuc }} .
$$

Here $U_{\text {nuc }}$ is the contribution from the molecular nuclei. Just as we need to average over all orientations to obtain the potential for the molecular electrons, we also need to average over all orientations for the nuclei. Averaging a nucleus over all orientations is equivalent to placing the nuclear charge on a spherical shell which has a radius equal to the distance from the nucleus to the center of mass. Consequently, $U_{\text {nuc }}$ is a sum of potentials for concentric spheres for each nucleus centered at the center of mass.

In addition to the static distorting potential, it is standard practice to add additional terms designed to approximate known important physical effects. Two such effects are exchange distortion $\left(U_{E}\right)$ (effect of continuum electron exchanging with passive electrons), and the correlationpolarization potential $U_{\mathrm{CP}}$

$$
U_{i}=U_{\text {static }}+U_{E}+U_{\mathrm{CP}} .
$$


For $U_{E}$, we use the exchange-distortion potential of Furness and McCarthy [190] (corrected for errors-see Riley and Truhlar [191]). In this approximation, the exchange potential $U_{E}$ depends on the average molecular charge density

$$
\begin{aligned}
& U_{E}\left(r_{a}\right) \\
& \quad=0.5\left[\varepsilon_{i}-U_{\text {static }}\left(r_{a}\right)-\sqrt{\left(\varepsilon_{i}-U_{\text {static }}\left(r_{a}\right)\right)^{2}+8 \pi \rho^{\text {ave }}\left(r_{a}\right)}\right] .
\end{aligned}
$$

One needs to be careful when looking at papers which use the Furness-McCarthy approximation since different definitions of the radial density are often used. For (23), the integral of the radial density over all space yields the number of electrons in the molecule. Frequently, a radial density is used for which the integral over radius only yields the number of electrons in the target (we used this definition in the past for atoms where the angular dependence is simply a spherical harmonic). The difference is replacing $8 \pi \rho^{\text {ave }}$ with $2 \rho^{\text {ave }}$ (see also, e.g., Martinez et al. [97]).

For the correlation-polarization potential $U_{\mathrm{CP}}$, we use the approximation of Perdew and Zunger [192] (see also Padial and Norcross [193]). The final state distorting potential $U_{\text {ion }}$ is calculated in the same way as $U_{i}$ except that the active electron is removed in the calculation of the charge density.

As mentioned in the introduction, including the full final state Coulomb interaction $\mathrm{C}_{\text {proj-eject }}$ in the wavefunction requires the evaluation of a numerical $6 \mathrm{D}$ integral. This factor is given by

$$
\mathrm{C}_{\text {proj-eject }}=e^{-\pi \gamma / 2} \Gamma(1-i \gamma){ }_{1} F_{1}\left(i \gamma, 1,-i\left(k_{a b} r_{a b}+\mathbf{k}_{a b} \cdot \mathbf{r}_{a b}\right)\right) \text {. }
$$

Here ${ }_{1} F_{1}$ is a confluent hypergeometric function, $\Gamma(1-i \gamma)$ is the gamma function, $\mathbf{k}_{a b}=\mu v_{a b}, \mu=1 / 2$ is the reduced mass for two electrons, $v_{a b}$ is the relative velocity between the two electron, and $\gamma$ is the Sommerfeld parameter $\gamma=1 / v_{a b}$ which is a measure of the strength of the coulomb interaction between the two electrons.

For the lower energies, it has become clear that using the full Coulomb interaction of (24) tends to overestimate the effect of the PCI. We have found that the low energy approximation of Ward and Macek [109] often yields very good agreement with experimental data. In the Ward-Macek approximation, one replaces the actual final state electronelectron separation $\mathbf{r}_{a b}$ by an average value directed parallel to $\mathbf{k}_{a b}$. The average separation is given by

$$
r_{a b}^{\mathrm{ave}}=\frac{\pi^{2}}{16 \varepsilon_{t}}\left(1+\frac{0.627}{\pi} \sqrt{\varepsilon_{t}} \ln \varepsilon_{t}\right)^{2},
$$

where $\varepsilon_{t}$ is the total energy of the scattered and ejected electrons. With this approximation, the $\mathrm{C}_{\text {proj-eject }}$ factor can be removed from the $T$-matrix integral which means that the computational difficulty reduces to that of the standard distorted-wave approximation. Since the cross section is proportional to the square of the $T$-matrix, in the WardMacek approximation the standard distorted wave cross sections are multiplied by

$$
\left|\mathrm{C}_{\text {proj-eject }}\right|^{2}=N_{e e}\left|{ }_{1} F_{1}\left(i \gamma, 1,-2 i k_{a b} r_{a b}^{\text {ave }}\right)\right|^{2},
$$

where $N_{e e}$, which called the Gamow factor, is defined as:

$$
N_{e e}=\left|e^{-\pi \gamma / 2} \Gamma(1-i \gamma)\right|^{2}=\frac{\pi / k_{a b}}{\left(e^{\pi / k_{a b}}-1\right)} .
$$

Botero and Macek [107] (see also Whelan et al. [31], and Rasch et al. [194]) proposed neglecting the hypergeometric function and just using the Gamow factor to approximate $\mathrm{C}_{\text {proj-eject. }}$ Both of these approximations allow one to factor the $\mathrm{C}_{\text {proj-eject }}$ term outside the integral which means that the computational difficulty is reduced to that of a DWBA calculation.

Finally, (17) is for the direct scattering amplitude. Since one cannot distinguish the projectile electron from the ejected electron, we have to evaluate the exchange amplitude as well

$$
\begin{gathered}
T_{\text {exc }}^{\mathrm{M} 3 \mathrm{DW}}=\left\langle\chi_{\text {proj }}\left(\mathbf{r}_{b}\right) \chi_{\text {eject }}\left(\mathbf{r}_{a}\right) \mathrm{C}_{\text {scat-eject }}\left(\mathbf{r}_{a b}\right)\right| \frac{1}{r_{a b}} \\
-U_{a}\left(r_{a}\right)\left|\psi_{\text {Dyson }}^{\text {OAMO }}\left(r_{a}\right) \chi_{i}\left(\mathbf{r}_{b}\right)\right\rangle .
\end{gathered}
$$

With these amplitudes, the fully differential cross-section for molecules can be obtained from

$$
\frac{d^{5} \sigma}{d \Omega_{a} d \Omega_{b} d E_{b}}=\frac{1}{(2 \pi)^{5}} \frac{k_{a} k_{b}}{k_{i}}\left(\left|T_{\mathrm{dir}}\right|^{2}+\left|T_{\mathrm{exc}}\right|^{2}+\left|T_{\mathrm{dir}}-T_{\mathrm{exc}}\right|^{2}\right) .
$$

\section{Results}

There have been numerous high and low energy experimental papers published over the last $20-30$ years examining electron-impact ionization of molecules with limited theoretical support. In the last few years, the level of theoretical support has increased and here we will give an overview of the current status of the agreement between experiment and theory. In particular, we will present the recent experimental and theoretical results for $\mathrm{H}_{2}, \mathrm{~N}_{2}, \mathrm{H}_{2} \mathrm{O}$, and formic acid (FA) or $(\mathrm{HCOOH})$. We would like to emphasize that we are not trying to provide an overview of all the low to intermediate energy work that has been done-only the work performed in the last few years.

3.1. $\mathrm{H}_{2}$. Two sets of experimental data have been recently reported for electron-impact ionization of $\mathrm{H}_{2}$. Azzedine Lahmam-Bennani's group in Paris, France has performed some measurements for relatively high energies and Andrew Murray's group at Manchester, England has reported low energy measurements. Figure 1 shows a comparison of the Paris data [170] with our M3DW results and the First Born Approximation-Two Center Continuum (FBA-TCC) calculation of Joulakian and co-workers. The cross-sections presented in Figure 1 exhibit the typical $(\mathrm{e}, 2 \mathrm{e})$ pattern normally found for ionization of atoms-a large peak at small scattering angles and a small peak at large scattering angles (normally at about $180^{\circ}$ from the large peak). The large peak is called the binary peak since it is typically fairly close to the angle one would calculate classically for a binary collision between the projectile and a second electron at rest. 


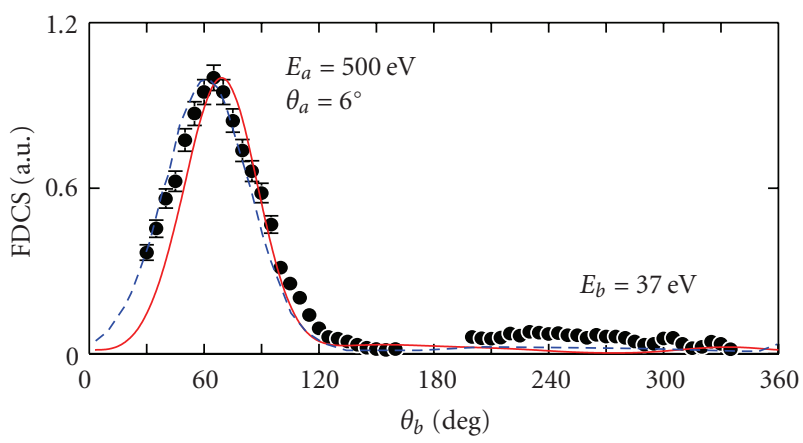

(a)

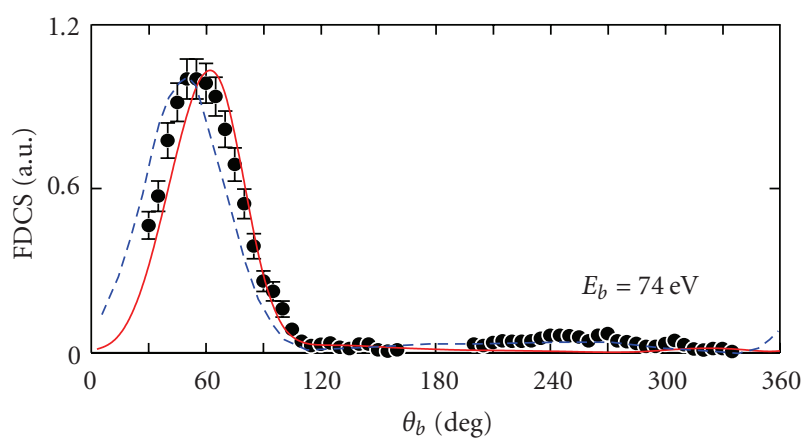

(b)

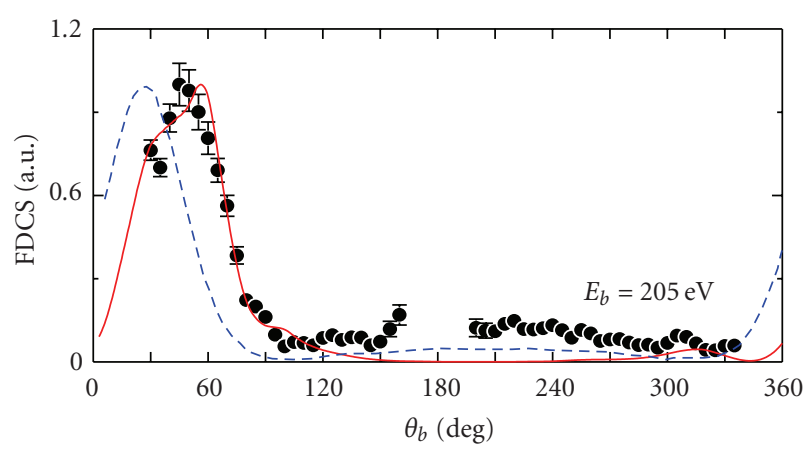

- $\mathrm{H}_{2}$ expt

- - FBA-TCC

- M3DW

(c)

FIGURE 1: FDCS for $\mathrm{H}_{2}$ as a function of the ejected electron angle. The scattered electron energy was $500 \mathrm{eV}$ and the scattering electron angle was $6^{\circ}$. The ejected electron energies were $37 \mathrm{eV}, 74 \mathrm{eV}$, and $205 \mathrm{eV}$ as shown in the figure. The experimental results are compared to M3DW calculations (solid line) and FBA-TCC (dashed line) and both normalized to unity at the experimental maximum.

The small peak (barely visible in these measurements plotted on a linear scale) is called the recoil peak and it is attributed to a double scattering mechanism-a binary collision for which the atomic electron is headed in the binary peak direction followed by a $180^{\circ}$ backscattering from the positive ion.

The M3DW results were calculated using the orientationaveraged molecular orbital (OAMO) approximation and, as mentioned in the theory section, this approximation is expected to be valid for highly symmetric molecular states and scattering angles for which the momentum transferred to the residual ion is small (less than unity). The momentum transferred to the ion is zero in the binary peak direction and small for angles in the binary peak. For the kinematics of the Paris data, the momentum transferred to the ion is quite large for angles in the recoil peak so the OAMO approximation is expected to be valid for the binary peak but not the recoil peak. Figure 1 presents results for which the projectile is scattered through an angle of $6^{\circ}$ with an energy of $500 \mathrm{eV}$. The horizontal axis is the detection angle for the ejected electron which has energies of 37, 74 and $205 \mathrm{eV}$ as shown. For the two lower energies, both theoretical approaches are in qualitative agreement with experiment although the binary peak position of the M3DW tends to be at a slightly higher angle than experiment while the FBATCC is at a somewhat smaller angle. For the highest energy, the M3DW is noticeably closer to the experimental binary peak and there also appears to be some structure in the data, which is predicted by the M3DW. On the other hand, the FBA-TCC is in somewhat better agreement with experiment for the recoil peak as might be expected since the OAMO approximation is not expected to be valid in this angular range.

Figure 2 presents a comparison between experiment and theory for the low-energy measurements from Manchester [177-179]. The Manchester experiment is unique in that the incident beam direction can be rotated relative to the detection plane for the two final-state electrons. The angle $\psi$ in Figure 2 is the angle between the beam direction and detection plane with $\psi=0^{\circ}$ being the scattering plane and $\psi=90^{\circ}$ being perpendicular to the incident-beam direction. For the data of Figure 2, both final state electrons have equal energies of $10 \mathrm{eV}$ and the horizontal axis is the angle between the two electrons. (It should be noted that the Manchester group normally report their data relative to an angle that is half the mutual detection angle, i.e., in Figure 2, back-toback scattering is $180^{\circ}$ whereas it would be $90^{\circ}$ in the original data set.)

Three theoretical results are presented in Figure 2-the time-dependent close coupling (TDCC) results of Colgan and co-workers and the M3DW both with and without the Perdew-Zunger correlation-polarization potential. Although the experimental data are not absolute, there is a common point for all 6 data sets which means that there is only one normalization for all the data sets and we have chosen to normalize experiment and theory in the perpendicular plane. With this normalization, it is seen that both the TDCC and the M3DW-CP are in quite good agreement with experiment in the perpendicular plane. The interesting and peculiar feature is that agreement between experiment and theory gets worse as the one approaches the scattering plane and, contrary to what one might expect, we get the worst agreement in the scattering plane. Of course, one could normalize in the scattering plane instead of the perpendicular plane, but then the overall agreement between experiment and theory would look significantly worse. With this normalization, the TDCC looks very good down to $\psi=$ $67^{\circ}$ and the M3DW looks reasonably good down to $\psi=45^{\circ}$. 


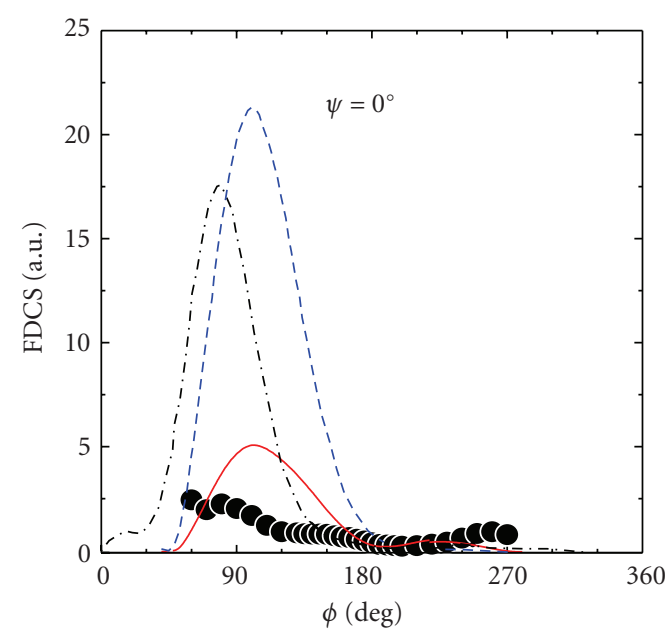

(a)

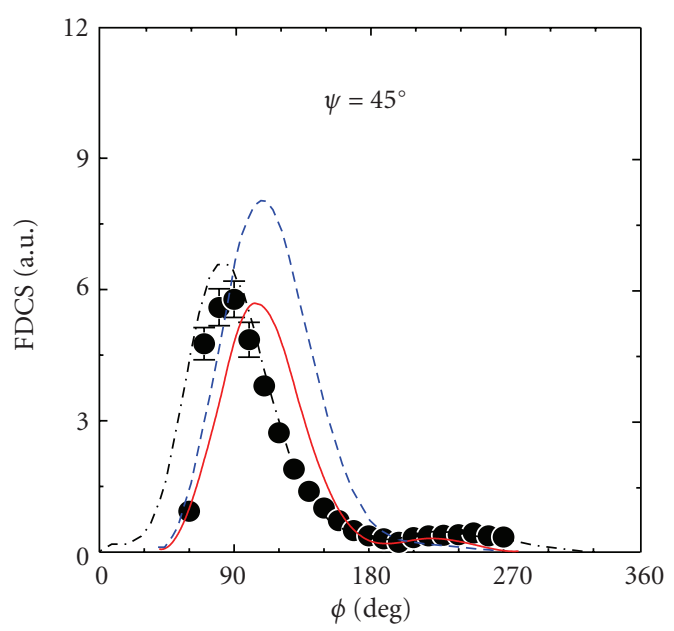

(c)

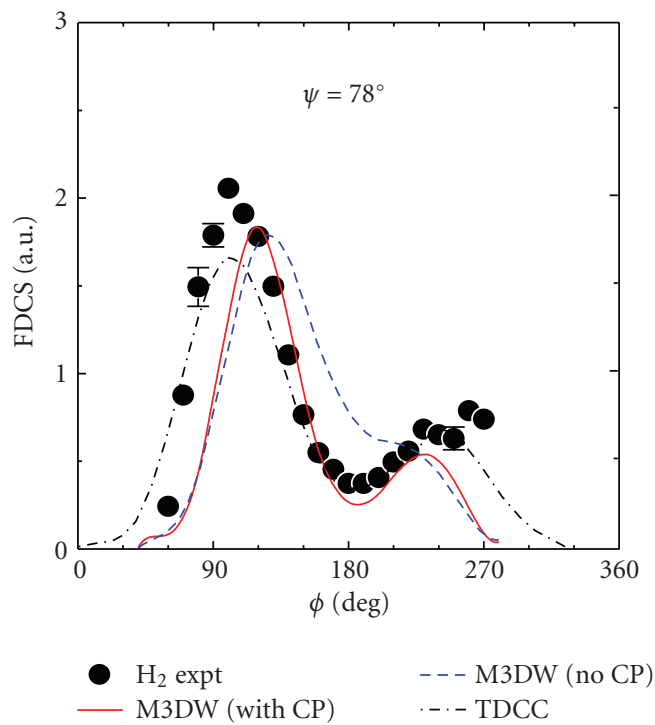

(e)

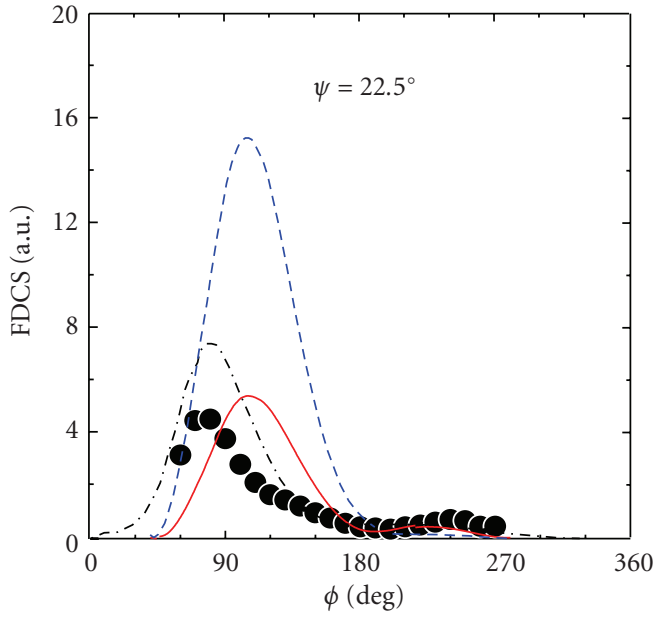

(b)

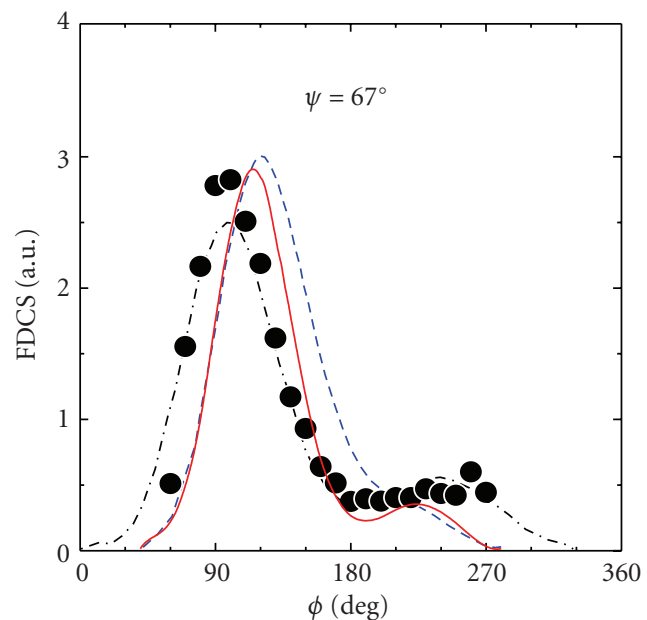

(d)

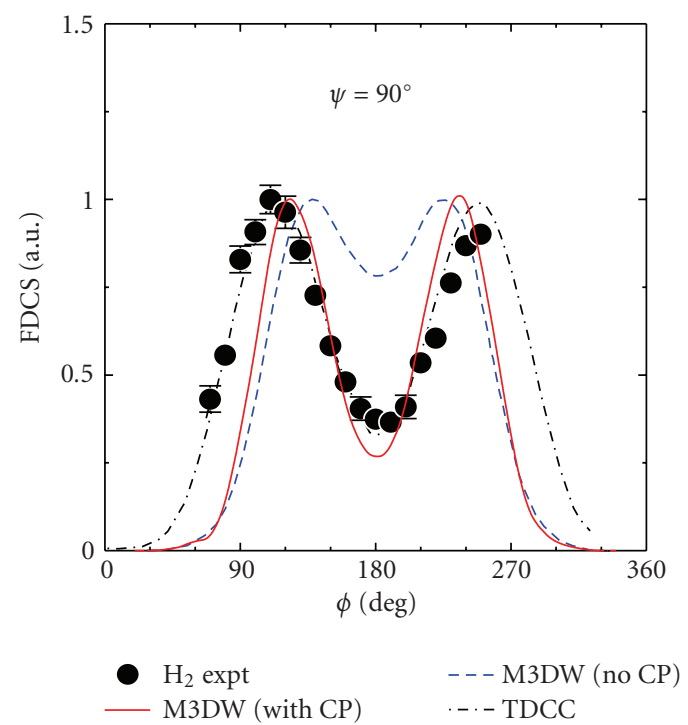

(f)

FIGURE 2: FDCS for electron impact ionization of $\mathrm{H}_{2}$ for equal final state energies $E_{a}=E_{b}=10 \mathrm{eV}$ with various values of the angle $\psi$ (the angle $\psi$ is the angle between the beam direction and detection plane) as a function of the angle between the outgoing electrons. The experiment results are compared with TDCC (dotted-dashed line), M3DW with CP (solid line), and M3DW with no CP (dashed line) calculations, where $\mathrm{CP}$ is correlation-polarization potential. The experimental and theoretical calculations are normalized to unity at the experimental maximum at $\psi=90^{\circ}$. 
One of the interesting aspects of the experimental data lies in the fact that the largest cross-section occurs for $\psi=45^{\circ}$ and not in the scattering plane. Only the M3DW$\mathrm{CP}$ agrees with this observation. Although the agreement between experiment and M3DW-CP for the location of the first peak position becomes worse for decreasing $\psi$, the height of this peak is predicted fairly well down to $\psi=22.5^{\circ}$. One cannot tell if this continues to be true in the scattering plane since the peak position is not determined by the data. One would certainly expect that the TDCC should be more accurate than the M3DW for these low energies and the success of the M3DW is quite surprising! Since adding the $\mathrm{CP}$ potential to the M3DW caused the theory to have a maximum for $\psi=45^{\circ}$, the TDCC calculation was repeated including this potential as well but a similar effect was not found.

3.2. $\mathrm{N}_{2}$. Low to intermediate energy experimental $(\mathrm{e}, 2 \mathrm{e})$ fully differential cross-section measurements for $\mathrm{N}_{2}$ have recently been made in three different laboratories-those of Azzedine Lahmam-Bennani (Paris, France), Andrew Murray (Manchester, England), and Birgit Lohmann (Adelaide, Australia). The higher energy experiments measured in Paris were able to resolve two features [170]. The first feature was direct ionization of the inner $2 \sigma_{g}$ state and the second feature contained ionization of three states-the $3 \sigma_{g}, 1 \pi_{u}$, and $2 \sigma_{u}$ which could not be resolved. As a result, to properly compare with experiment, a weighted sum of the theoretical results should be performed for the second feature. While this was possible for the FBA-TCC model, it was not possible for the M3DW since the OAMO approximation is not valid for the $1 \pi_{u}$ and $2 \sigma_{u}$ states.

Figure 3 compares the two theoretical calculations with the Paris FDCS measurements. For both cases, the incident energy was adjusted so that the faster final state electron had an energy of $500 \mathrm{eV}$ and the slower one had $74 \mathrm{eV}$ and the scattering angle for the faster one was $6^{\circ}$. There are two M3DW curves presented on Figure 3. The dash-dot curve is the original calculation contained in the publication of Naja et al. [170] which used Dyson orbitals calculated using GAMESS with a small basis set. The solid curve is a new calculation using Dyson orbitals calculated by C. G. Ning using density function theory with a B3LYP/TZ2P basis set. For the experimental $\left(3 \sigma_{g}, 1 \pi_{u}, 2 \sigma_{u}\right)$ state in Figure $3(\mathrm{~b})$, the new results ( $3 \sigma_{g}$ only) are in better agreement with the binary peak (and also in better agreement with the FBATCC results). For the $2 \sigma_{g}$ state in Figure $3(\mathrm{a})$, the new results predict a double binary peak. Although the experimental data suggest the possibility of a double binary peak, the experimental peak positions are shifted from the theoretical calculations. In fact, the different theoretical predictions for the binary peak positions agree better with each other than with experiment! Compared to $\mathrm{H}_{2}$, the experimental recoil peak is relatively much stronger (as big as the binary peak for the $2 \sigma_{g}$ state). While the FBA-TCC prediction for the $\left(3 \sigma_{g}, 1 \pi_{u}, 2 \sigma_{u}\right)$ recoil lobe is in qualitative agreement with experiment, the M3DW predictions for the recoil lobe are much different from the experiment. For the $2 \sigma_{g}$ state, none

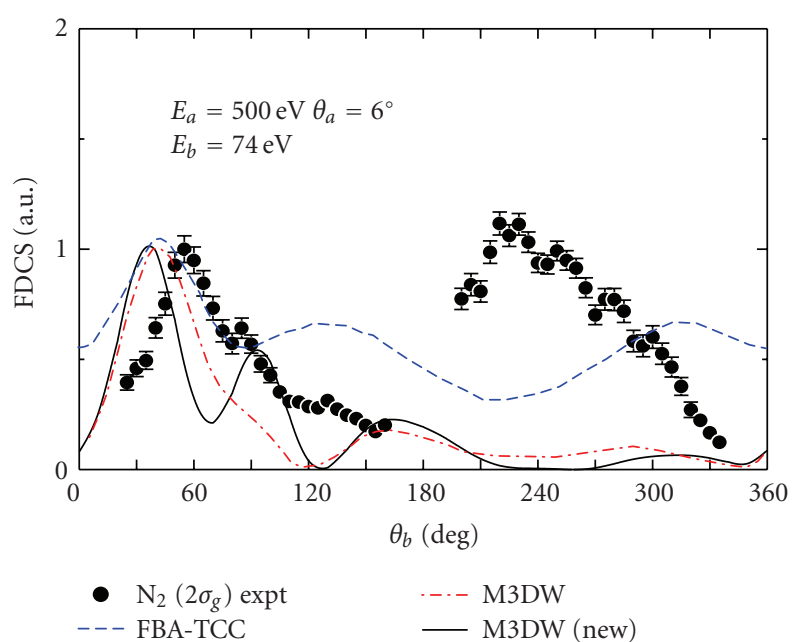

(a)

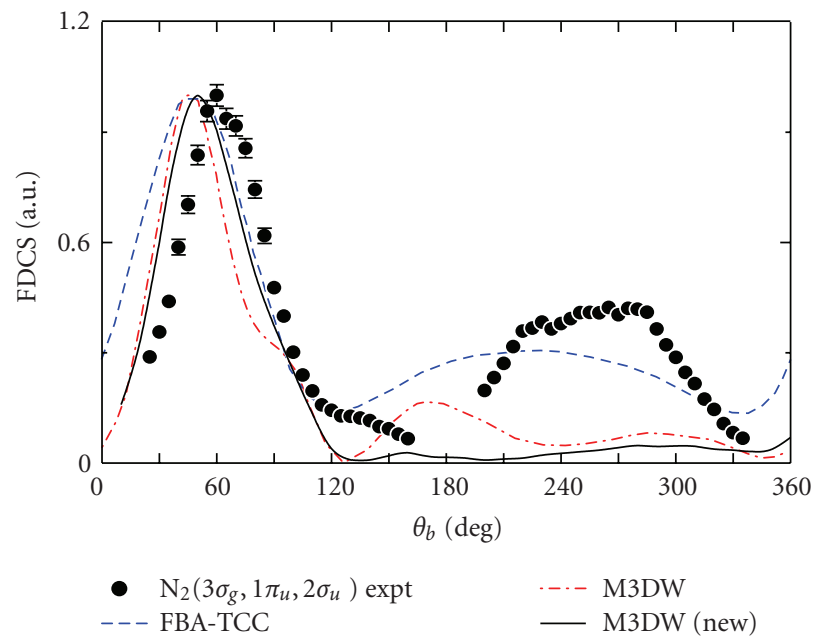

(b)

FIGURE 3: FDCS for electron-impact ionization of $\mathrm{N}_{2}$ for the $2 \sigma_{g}$ orbital (a) and for sum of $3 \sigma_{g}, 1 \pi_{u}$, and $2 \sigma_{g}$ orbitals (b). The scattered electron energy was $500 \mathrm{eV}$ and the scattering angle was $6^{\circ}$. The ejected electron energy was $74 \mathrm{eV}$. The experimental results are compared with the FBA-TCC (dashed line) and two sets of M3DW calculations. The dotted-dashed line is the M3DW using a poor wave function and the solid line is the M3DW using an improved wave function. The experimental and theoretical data are normalized to the experimental binary peak.

of the theoretical predictions resembles the recoil peak found in the experimental data.

The experimental measurements performed at Manchester and Adelaide were for lower incident-electron energies. In both cases, the $3 \sigma_{g}, 1 \pi_{u}$, and $2 \sigma_{u}$ states could be resolved. Here we present a comparison between experiment and the M3DW theory for the $3 \sigma_{g}$ state only in Figure 4 for incident electron energies of $75 \mathrm{eV}$ and $150 \mathrm{eV}$. To date, no other molecular calculation has been reported for these energies for $\mathrm{N}_{2}$ to our knowledge except using the Stia et al. [149] approximation in which molecular cross-sections for 


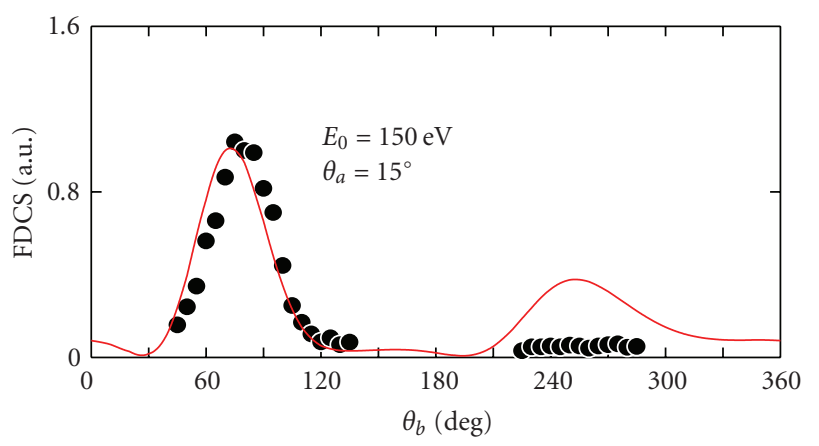

- $\mathrm{N}_{2} \operatorname{expt}$ - M3DW

(a)

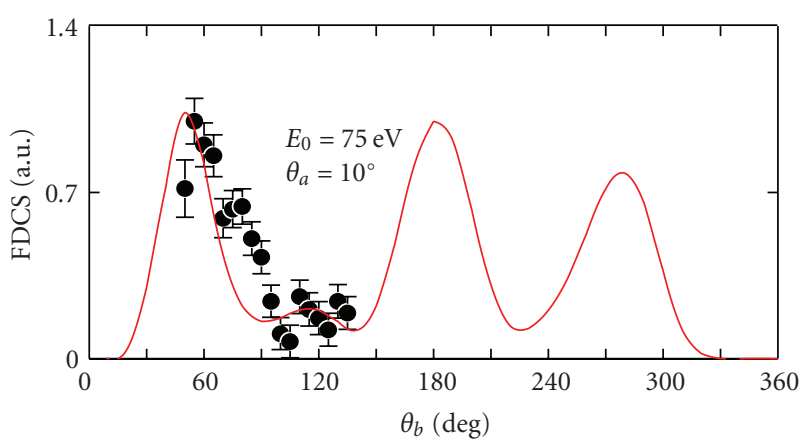

- $\mathrm{N}_{2} \operatorname{expt}$

M3DW

(b)

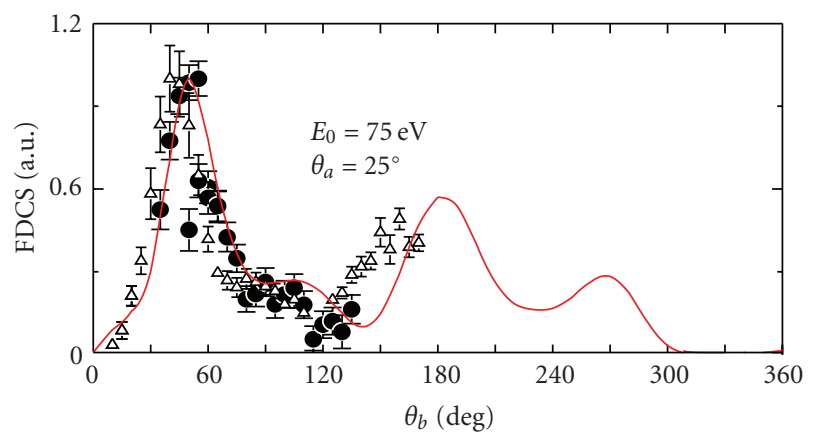

$\triangle$ Manchester $\left(22^{\circ}\right)$

- Adelaide $\left(25^{\circ}\right)$

(c)

FIGURE 4: FDCS for electron-impact ionization of $3 \sigma_{g}$ orbital of $\mathrm{N}_{2}$. The incident electron energy for (a) was $150 \mathrm{eV}$, the ejected electron energy was $10 \mathrm{eV}$, and the projectile scattering angle was $15^{\circ}$. For (b) and (c), the incident electron energy was $75 \mathrm{eV}$, the scattered and the ejected electron energies were $30 \mathrm{eV}$, and the scattering angle for one of the electrons was fixed at $10^{\circ}$ and $25^{\circ}$ as shown in the figure. The Adelaide (circles) and Manchester (triangles) experiment results are compared to M3DW calculations (solid curve). The experimental and theoretical data are normalized to unity at the experimental maximum.

diatomic molecules are approximated as the atomic crosssection times and an interference factor. As can be seen from Figure 4, the M3DW results are in quite good agreement with the experimental binary peaks. For the lower energy in Figures 4(b) and 4(c), both the Manchester and Adelaide data exhibit some additional structure in the $80^{\circ}-120^{\circ}$ angular range which is also predicted by the M3DW. It is difficult and time consuming to measure data in the angular range of the recoil peak and this has been done at Adelaide only for the higher energy. Consistent with the $\mathrm{H}_{2}$ results, the agreement between experiment and the M3DW theory is not very good for the recoil peak. Interestingly, the Stia et al. [149] approximation of multiplying the atomic cross-section times an interference factor yields quite good agreement with the recoil data although the binary peak is not as good as found for the M3DW. Hargreaves et al. [195] interpreted this as evidence for a Young's type interference for electron scattering from a diatomic molecule which will be discussed below.

For $75 \mathrm{eV}$ incident electrons, the M3DW predicts a significant peak at $180^{\circ}$ backscattering (i.e., in the incident beam direction) and the existing experimental data indicates that there is definitely a peak near that direction. The prediction is that the size of this peak will grow with decreasing projectile scattering angle as can be seen from Figures 4(b) and 4(c). Gao et al. [158] interpreted this peak as a Young's type interference for electrons backscattered from two different nuclei. However, if this were the case, the peak should vanish if the separation between the nuclei was reduced to zero. Hargreaves et al. [195] presented M3DW results for which the separation between the nuclei was reduced to zero and the $180^{\circ}$ peak did not go away. Consequently, it is clear that this peak does result from some sort of quantum mechanical interference but we do not believe that it is a Young's type of interference.

3.3. $\mathrm{H}_{2} \mathrm{O}$. Fully differential cross-sections for electronimpaction ionization of $\mathrm{H}_{2} \mathrm{O}$ have been recently measured at both Adelaide and Manchester. Milne-Brownlie et al. [155] reported FDCS measurements for the summed $3 a_{1}+$ $1 b_{1}$ states for $250 \mathrm{eV}$ incident electrons, Kaiser et al. [168] reported results for the $1 b_{1}$ state for a range of different kinematics with incident electron energies varying between $30 \mathrm{eV}$ and $110 \mathrm{eV}$, and Nixon et al. [196] measured low energy $3 a_{1}$ FDCS (energy above threshold varying between $4 \mathrm{eV}$ to $40 \mathrm{eV}$ ). Although Kaiser et al. [168] showed M3DW results for the $1 b_{1}$ state, we now know that the OAMO approximation is not valid for this state even for the binary peak so we will not show those results here. On the other hand, the OAMO approximation should be reasonable good for the $3 a_{1}$ state. Figure 5 contains a comparison between the M3DW results and the Nixon et al. [196] measurements for the FDCS for ionization of the $3 a_{1}$ state. Similar to the $\mathrm{H}_{2}$ measurements presented in Figure 2, $\psi$ is the angle between the incident beam direction and the detection plane for the two electrons. For the results of Figure 5, both final state electrons have an energy of $10 \mathrm{eV}$ and the horizontal axis is the angle between the two final state electrons in the detection plane. As before, only one normalization is used for all 5 sets of data. The only theoretical results available are the M3DW and, for Figure 5 we have shown results for the 


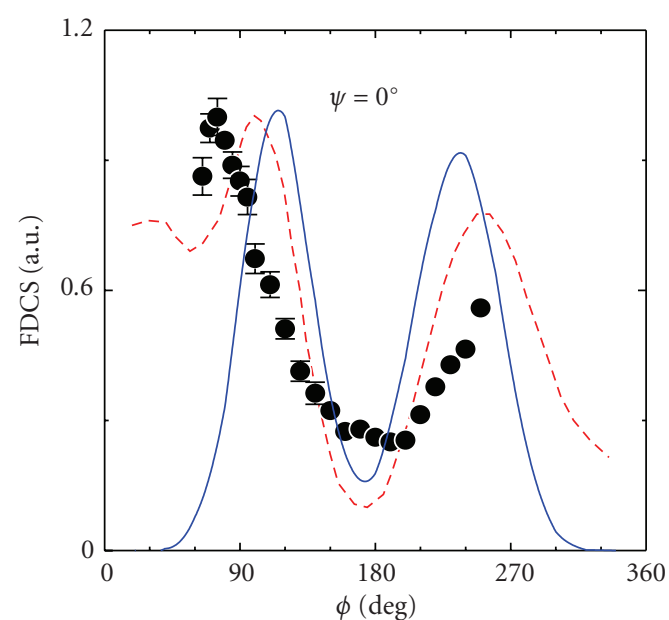

(a)

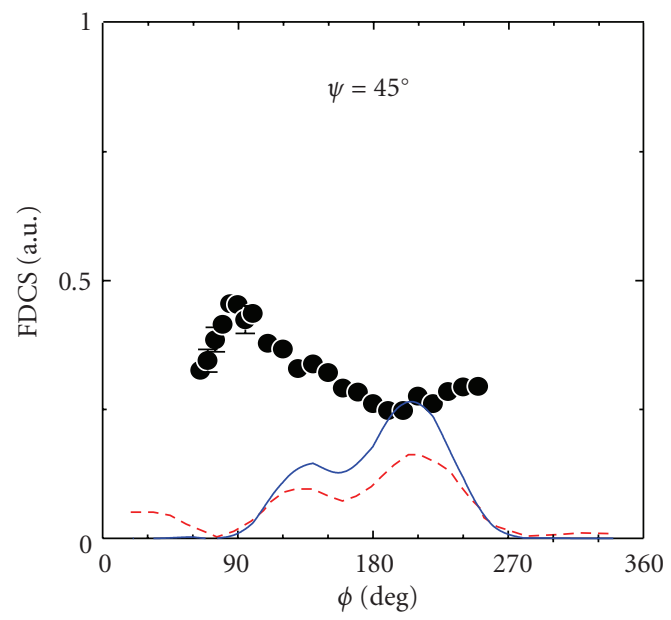

(c)

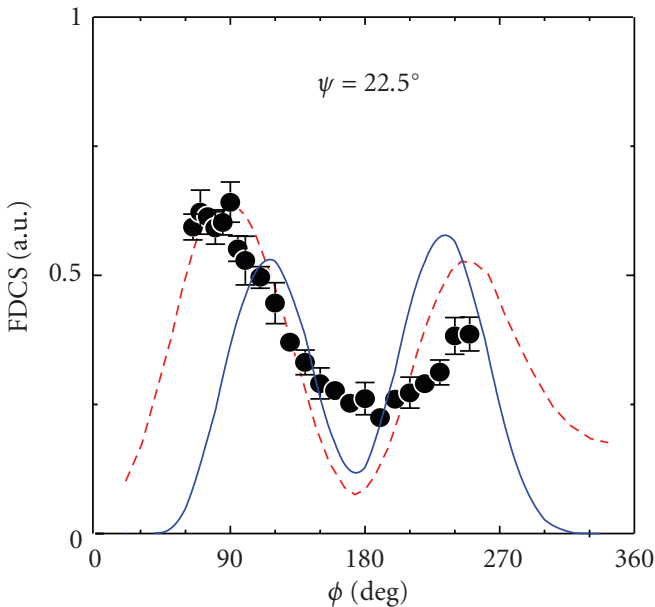

(b)

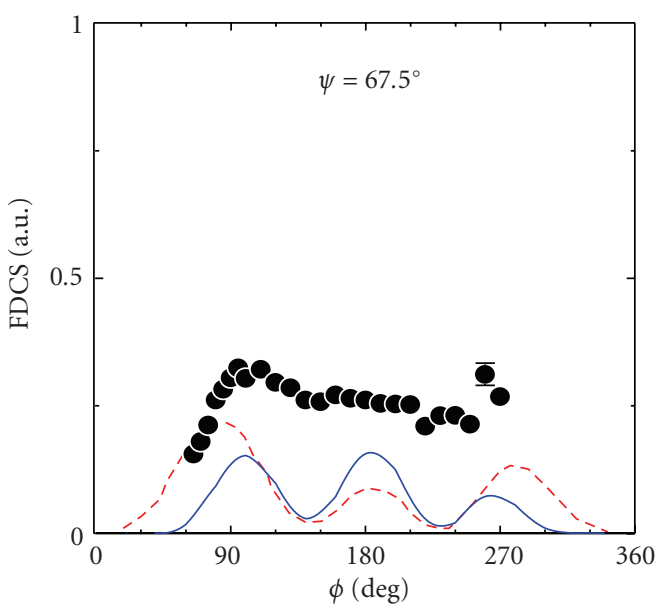

(d)

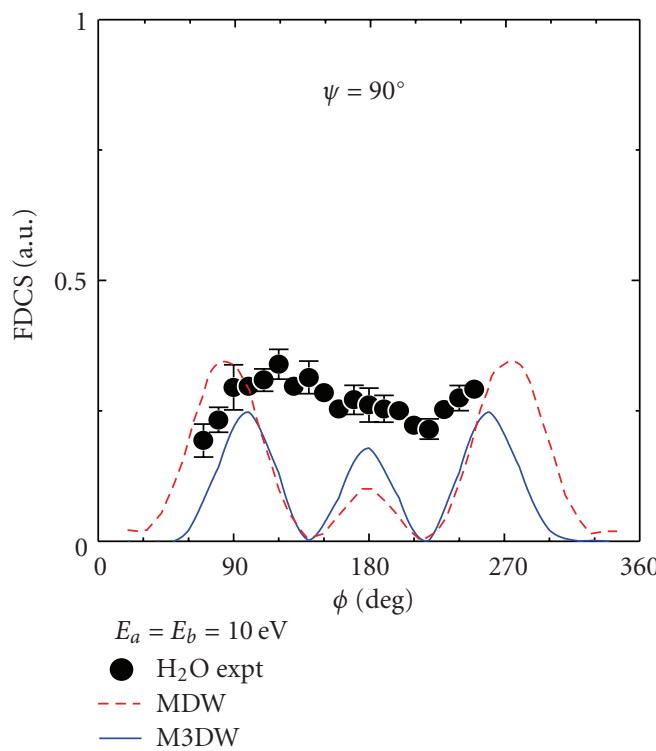

(e)

FIgURE 5: FDCS for electron-impact the ionization of the $3 \mathrm{a}_{1}$ state of $\mathrm{H}_{2} \mathrm{O}$ for equal final state energies $E_{a}=E_{b}=10 \mathrm{eV}$ with various values of the angle $\psi$ (the angle $\psi$ is the angle between the beam direction and detection plane) as a function of the angle between the outgoing electrons. The experiment results are compared with M3DW (solid line) and MDW (dashed line) calculations. The experimental and theoretical calculations are normalized to unity at the peak for $\psi=0^{\circ}$. 
standard DWBA (MDW of (8)) as well as the M3DW. The only difference between the two calculations is the Coulomb interaction in the final state wave function (see (11)).

Figure 5 results are completely opposite from the $\mathrm{H}_{2}$ results of Figure 2. For $\mathrm{H}_{2}$, the best agreement between experiment and theory was in the perpendicular plane and here we see the best agreement between experiment and theory is in the scattering plane and the worst agreement is in the perpendicular plane. For $\mathrm{H}_{2}$, the largest crosssection was found for $\psi=45^{\circ}$ and here the largest cross-section is in the scattering plane (both experiment and theory). The smallest experimental cross-section lies in the perpendicular plane whereas the smallest theoretical cross-section was found for intermediate $\psi$ angles. In terms of agreement between experiment and theory, the MDW (standard DWBA) predicts the maxima in the cross-section overall better than the M3DW which is hard to understand.

The most puzzling aspect of Figure 5 lies in the perpendicular plane. Al-Hagan et al. [110] examined the behavior of the FDCS in the perpendicular plane for atoms and molecules. In that paper, it was predicted that the FDCS in the perpendicular plane should have a maxima for $90^{\circ}$ and $270^{\circ}$ scattering for all molecules (and atoms). It was further predicted that molecules which have no nucleus at the center of mass (e.g., like a diatomic) would have a minimum in the FDCS for $180^{\circ}$ (back-to-back) scattering. Also, it was predicted that any molecule that has a nucleus at (or near) the center of mass should have a maximum in the FDCS for $180^{\circ}$ scattering. These predictions were experimentally verified for $\mathrm{H}_{2}, \mathrm{~N}_{2}$, and $\mathrm{CO}_{2}$. Here we see that the theoretical results are consistent with the Al-Hagan et al. [110] predictions. Although one might imagine a hint of a $180^{\circ}$ maximum in the experimental data, the data has very little structure between $90^{\circ}$ and $270^{\circ}$. The most optimistic scenario might be that the three peaks have merged together but there is no indication of this in the data for the intermediate $\psi$ angles. Consequently, this is currently an interesting unsolved puzzle.

3.4. Formic Acid. Finally, we looked at the biomolecule formic acid (FA) (HCOOH). Very recently, Colyer et al. [197] measured FDCS for electron impact ionization of the outer valence orbitals of FA by $100 \mathrm{eV}$ and $250 \mathrm{eV}$ incident electrons. The experiments were performed under asymmetric kinematics, in which the outgoing ejected electron had an energy of $10 \mathrm{eV}$. Unfortunately, the Adelaide experiment could not resolve the $10 a^{\prime}$ and $2 a^{\prime \prime}$ valence orbitals. The OAMO approximation is expected to be good for the $10 a^{\prime}$ orbital but not the $2 a^{\prime \prime}$ orbital. Consequently, it is not presently possible to make a clean comparison between the M3DW and the experiment.

There have been several high energy EMS studies performed for FA with the most recent being a study of seven of the highest occupied orbitals including the $10 a^{\prime}$ highest occupied molecular orbital (HOMO) by Nixon et al. [198]. Each of the seven orbitals could be resolved and the experimental momentum distributions were compared with PWIA theoretical predictions obtained from wave functions

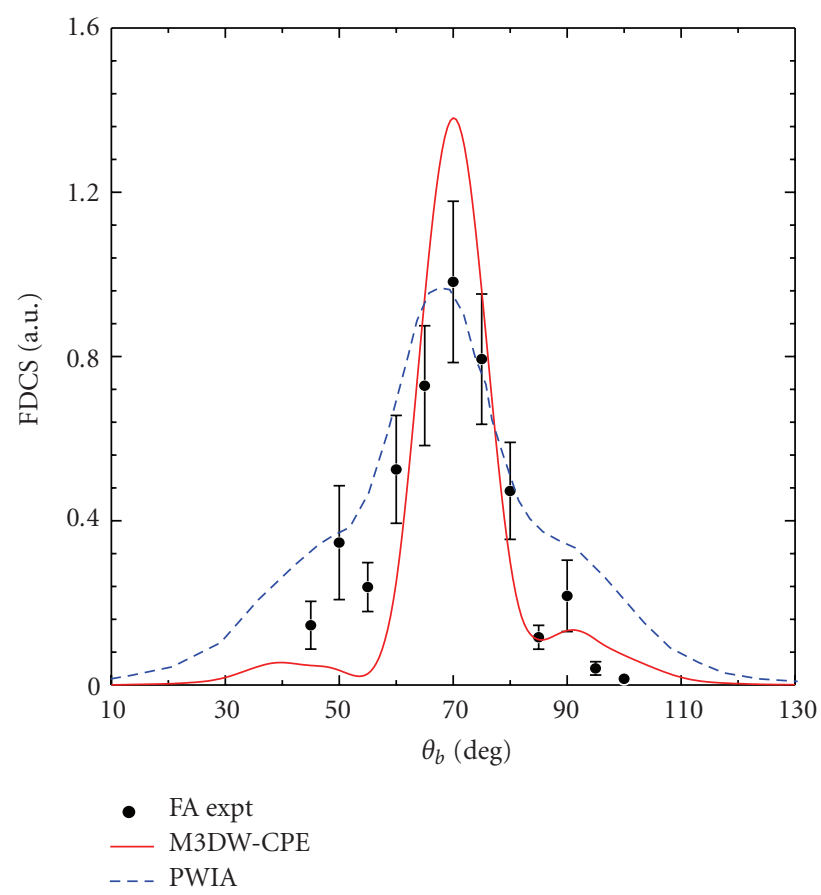

Figure 6: Theoretical and experimental FDCS for electron-impact ionization of the $10 a^{\prime}$ orbital of formic acid as a function of the ejected electron scattering angle. The incident electron energy was $831.6 \mathrm{eV}$, the fixed scattered projectile angle was $20.5^{\circ}$, and the ejected electron energy was $105 \mathrm{eV}$. The experiment results (circle) are compared to M3DW calculations (solid line) and PWIA calculations (dashed line).

calculated using density function theory with a B3LYP/TZP basis set. The OAMO wave functions we are using were calculated using a B3LYP/TZ2P basis set which should be essentially equivalent for our purposes. Since the EMS studies are designed to be a direct check of the quality of molecular wave functions, we thought it would be instructive to use the EMS data to check the quality of our OAMO wave function for the $10 a^{\prime}$ state. Figure 6 compares the Nixon et al. [198] results with our M3DW-CPE (M3DW including correlation-polarization and Furness-McCarthy exchange) for the $10 a^{\prime}$ state. For the Nixon's experiment, the incident electron energy is $831.6 \mathrm{eV}$, the projectile scattering angle is $20.5^{\circ}$, and the ejected electron energy is $105 \mathrm{eV}$.

The EMS theoretical results presented in the figure from the Nixon et al. [198] paper are based upon the plane wave impulse approximation (PWIA) of Weigold and McCarthy [138]. In the calculation of the PWIA, a proper average over molecular orientations is performed without making approximations. Consequently, the important difference between the PWIA and M3DW is the effects of the OAMO approximation plus the difference between using the plane wave impulse approximation and the distorted-wave Born approximation. Arguably, the M3DW-CPE results are in better overall agreement with the experimental data which would be understandable from the point of view that if kinematics are important, the DWBA should be better than the PWIA and $832 \mathrm{eV}$ is low enough an energy that 
kinematics might start playing a role. However, for this to be true, the OAMO approximation would also have to be valid. Consequently, the good agreement between the M3DW-CPE and the high energy experiment shown in Figure 6 indicates that the OAMO approximation is reasonably good for the $10 a^{\prime}$ state.

Figure 7 contains a comparison between the theoretical calculation for the $10 a^{\prime}$ state only and the Adelaide data, which is summed over the $10 a^{\prime}+2 a^{\prime \prime}$ states. Figures 7(a) and 7 (b) are for an incident electron energy of $100 \mathrm{eV}$ and projectile scattering angles of $10^{\circ}$ and $15^{\circ}$, respectively. Figures $7(\mathrm{c})$ and $7(\mathrm{~d})$ are for $250 \mathrm{eV}$ incident electrons and the same projectile scattering angles. The two theoretical results are M3DW both including and excluding correlationpolarization and exchange. Unlike the previous cases we have examined, there is not even qualitative agreement between experiment and theory for the binary peak. Since we have seen relatively good agreement with the binary peak for $830 \mathrm{eV}$ in Figure 6, we would assume that the lack of agreement seen here is primarily due to the $2 a^{\prime \prime}$ contribution, which is not included in the theory. It is interesting to note that the M3DW (which would be expected to be inferior) predicts the shape of the recoil peak better than the M3DW-CPE. However, this is probably fortuitous since the theory does not contain the $2 a^{\prime \prime}$ contribution plus the fact that we do not expect the M3DW with the OAMO approximation to yield accurate recoil peaks anyway. It is also interesting to note that the M3DW-CPE results for $250 \mathrm{eV}$ and $15^{\circ}$ scattering are in reasonable good agreement with the experimental data. Perhaps this indicates that the $2 a^{\prime \prime}$ state does not make a significant contribution for these kinematics?

3.5. Interference. There has been considerable interest in whether a Young's type double-slit interference effect could be seen for scattering from diatomic molecules. Stia et al. [149] predicted that, similar to photon scattering, the molecular scattering cross-section for $\mathrm{H}_{2}$ could be expressed as the atomic cross-section multiplied by an "interference" factor. The interference factor depends on the molecular separation and the momentum transferred to the residual ion. As a result, the shape of the molecular FDCS was predicted to be different from the shape of the atomic FDCS as modified by the interference factor.

Milne-Brownlie et al. [166] compared atomic and molecular $\mathrm{H}_{2}$ FDCS. For the cases they examined, the interference factor predicted that, relative to the binary peak, the molecular recoil peak should be smaller than the atomic recoil peak and this was verified by the experimental data. Consequently, this was interpreted as an observation of double-slit interference effects. A similar study was performed by Staicu-Casagrande et al. [175] for higher incident electron energies. The important aspect of this work was that three different ejected electron energies were studied and, for two of them, the interference factor predicted a reduction in the recoil peak while for one of them the prediction was for an enhancement and this prediction was also experimentally verified. Again, this was strong evidence supporting the observation of two-center effects. Very recently, Hargreaves et al. [195] performed a similar study for ionization of $\mathrm{N}_{2}$. Although the Stia et al. [149] prediction was for $\mathrm{H}_{2}$, it was assumed that the same idea would be applicable for any diatomic molecule. For the three cases considered, the interference factor predicted a reduction in the molecular recoil peak relative to the atomic case and very good agreement between experiment and theory was found using DWBA atomic cross-sections giving further evidence for two-slit effects.

3.6. Strength of a Perturbation Calculation. As mentioned in the introduction, there are now numerical techniques which can yield accurate FDCS for single ionization of atomic hydrogen, helium, and more recently molecular hydrogen. In terms of calculating reliable cross-sections, these methods should be clearly superior and preferable to any perturbation technique. Perturbation techniques such as the DWBA are expected to be valid for higher energies and we are amazed (and obviously pleased) at the accuracy of these techniques for the low energy results presented here. On the other hand, these perturbation techniques have some important advantages over numerical techniques. Even though the 6D integrals required for the M3DW are numerically intensive, they are still faster than the numerical methods particularly near threshold. Secondly, the M3DW can be applied to atoms or molecules of any size with equal ease. It is likely to be a very long time before numerical methods will be developed which can treat ionization of FA! Of course, the important question concerns the accuracy of the M3DW predictions and that is still an unanswered question. Finally, and most importantly, one of the great strengths of a perturbation calculation lies in the fact that one can easily investigate physical effects causing observed phenomena.

For example, Al-Hagan et al. [110] presented a comparison between ionization of $\mathrm{He}$ and $\mathrm{H}_{2}$ as shown in Figure 8. These are FDCSs measured in the perpendicular plane at Manchester, and the incident beams are adjusted so that the two final state electrons both have $10 \mathrm{eV}$ for both targets. The M3DW theoretical results are clearly in very nice agreement with the experiment for both cases. Comparing the equivalent molecular and atomic cases (i.e., same number of protons and electrons in the target), one immediately notices that both targets $\left(\mathrm{H}_{2}\right.$ and $\left.\mathrm{He}\right)$ have peaks around $90^{\circ}$ and $270^{\circ}$. On the other hand, He has the largest cross-section at $180^{\circ}$ while $\mathrm{H}_{2}$ has a deep minimum at $180^{\circ}$. The interesting question then concerns why the atomic and molecular targets behave similarly in some cases and completely opposite for other cases and these are the kind of questions that are well suited to a perturbation approach. For example, the atomic He data had been measured almost 20 years ago and Zhang et al. [16] showed how perturbation series can identify the physics leading to the $90^{\circ}$ and $270^{\circ}$ peaks. As mentioned in the theory section, the fundamental physics contained in a distorted-wave is elastic scattering from the target. A plane wave contains no elastic scattering from the target, and Zhang et al. [16] showed that the $90^{\circ}$ and $270^{\circ}$ peaks for $\mathrm{He}$ went away if the distorted-waves 


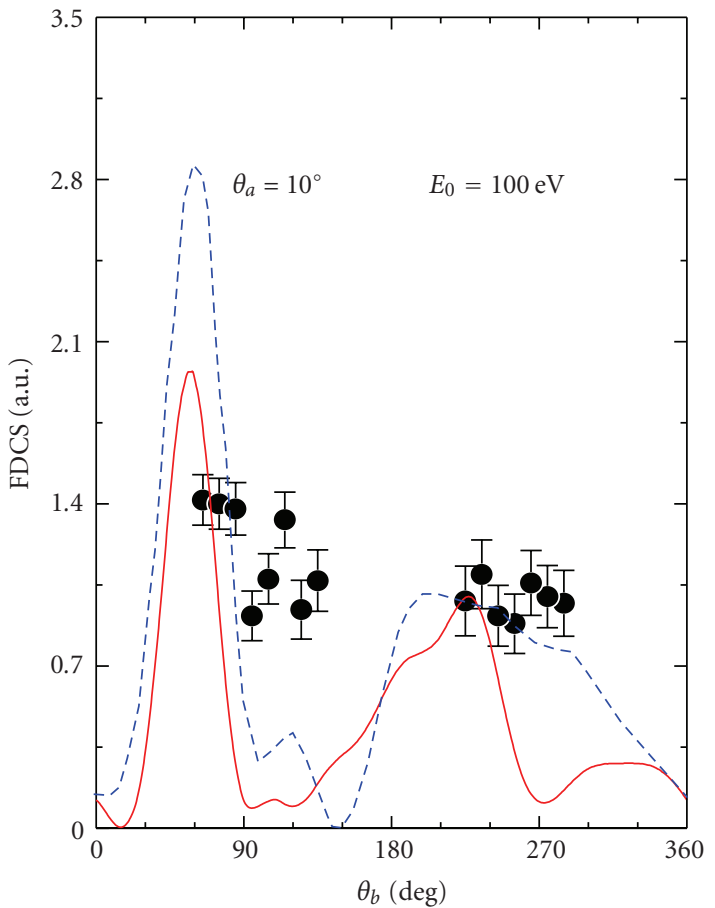

(a)

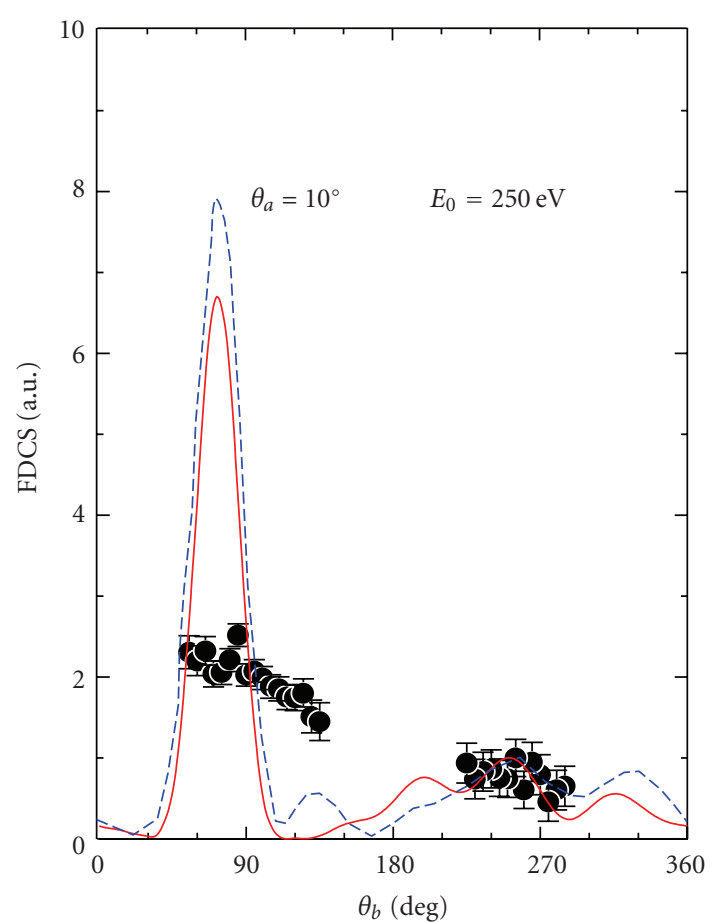

- FA expt

- - M3DW

M3DW-CPE

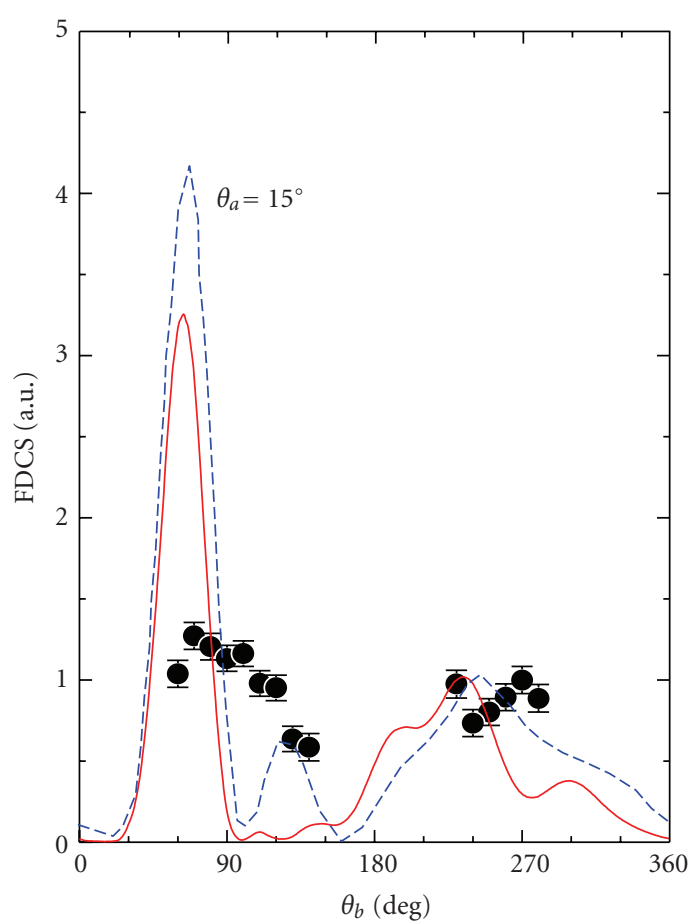

(b)

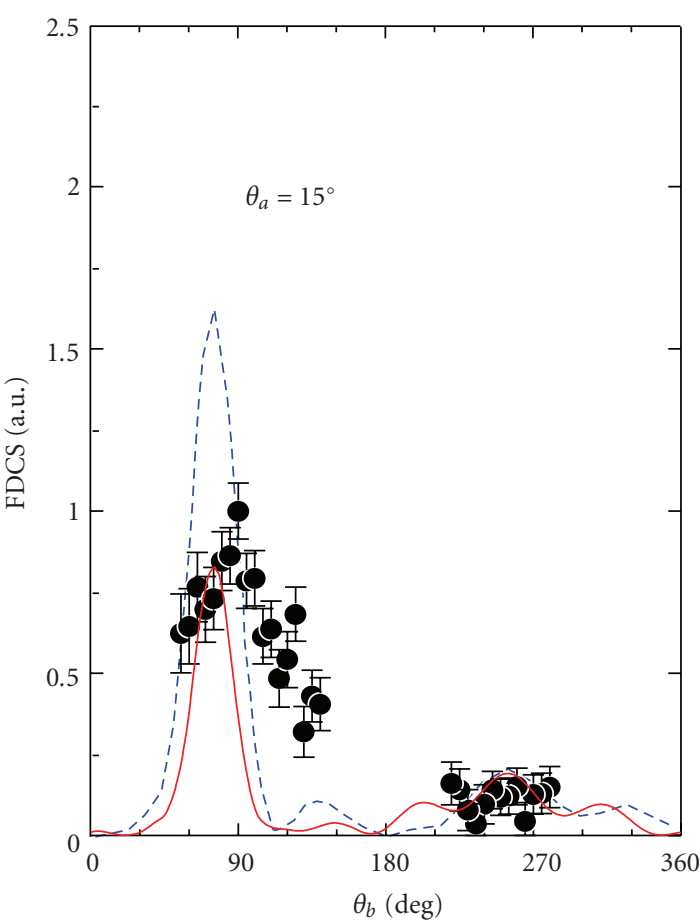

- FA expt

- - M3DW

M3DW-CPE

(c)

(d)

FIGURE 7: Experimental FDCSs for the sum of the $10 a^{\prime}+2 a^{\prime \prime}$ orbitals of formic acid as a function of the ejected electron angle, where (a) and (b) have $100 \mathrm{eV}$ incident energy electrons and (c) and (d) have $250 \mathrm{eV}$ incident energy electrons. The ejected electron energy is $10 \mathrm{eV}$ for all cases. The scattered electron angles are $10^{\circ}$ (for (a) and (c)) and $15^{\circ}$ (for (b) and (d)). The experiment results are compared with M3DW (dashed line) and M3DW-CPE (solid line) calculations for the $10 a^{\prime}$ orbital only. The experimental and theoretical data are normalized to the recoil peak. 


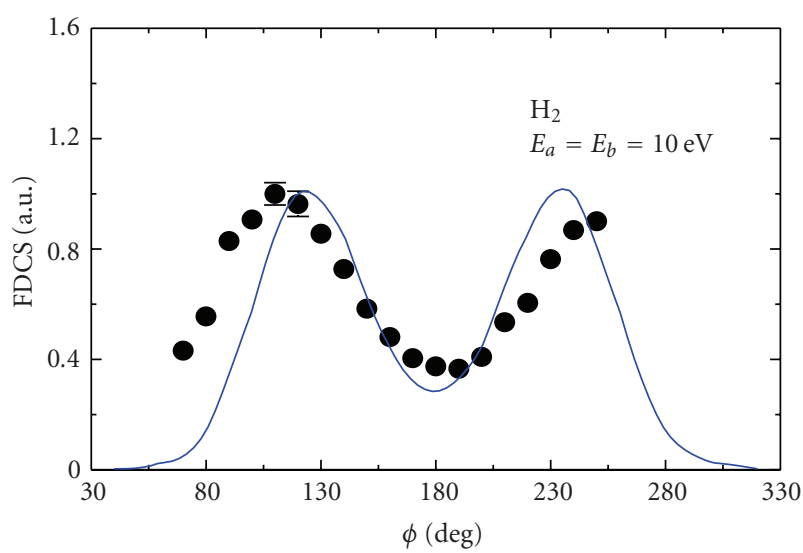

(a)

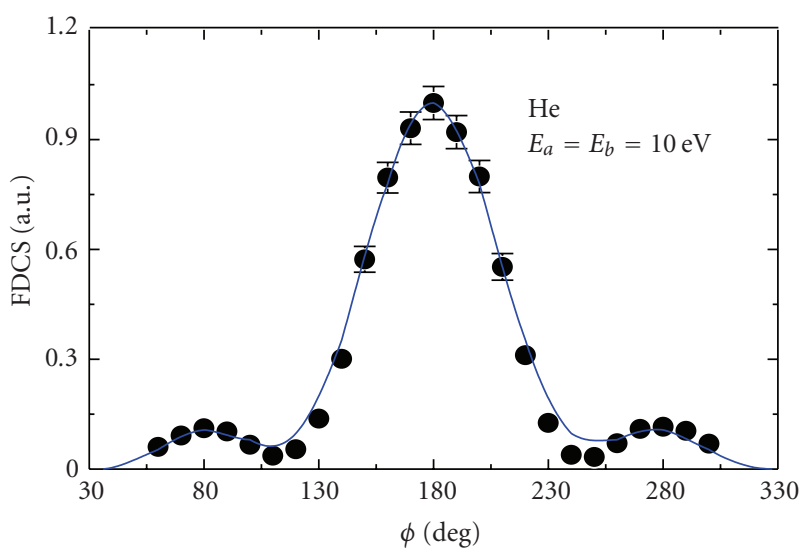

(b)

Figure 8: Experimental and theoretical FDCS for electron-impact ionization of $\mathrm{H}_{2}$ and $\mathrm{He}$ targets in the perpendicular plane. The outgoing electron energies were $E_{a}=E_{b}=10 \mathrm{eV}$ in both cases. The experimental results are compared with M3DW (solid line) and experiment and theory are normalized to unity at the experimental maximum.

were replaced with plane waves (i.e., if there was no elastic scattering from the target). Since $90^{\circ}$ and $270^{\circ}$ would be the mutual scattering angles for a classical collision between two equal mass particles with equal energies, the Zhang et al. [16] observation suggested that the physical effects leading to the $90^{\circ}$ and $270^{\circ}$ peaks were first elastic scattering from the target followed by a classical binary collision occurring in the perpendicular plane. Figure 9 contains the same type of comparison for $\mathrm{He}$ and $\mathrm{H}_{2}$ and it is seen that the $90^{\circ}$ and $270^{\circ}$ peaks go away for both targets without elastic scattering from the target. Consequently, the physics leading to the $90^{\circ}$ and $270^{\circ}$ peaks is the same for both the atomic and molecular targets.

The next question concerns why there is a maximum at $180^{\circ}$ for $\mathrm{He}$ and a minimum for $\mathrm{H}_{2}$. One of the important $(e, 2 e)$ mechanisms is backscattering from the nucleus so we decided to investigate the effect of the nuclear charge distributions on the FDCS. Since the distorting potential is a sum of the electronic part plus the nuclear part, one thing we can easily do is to leave the electronic part alone and
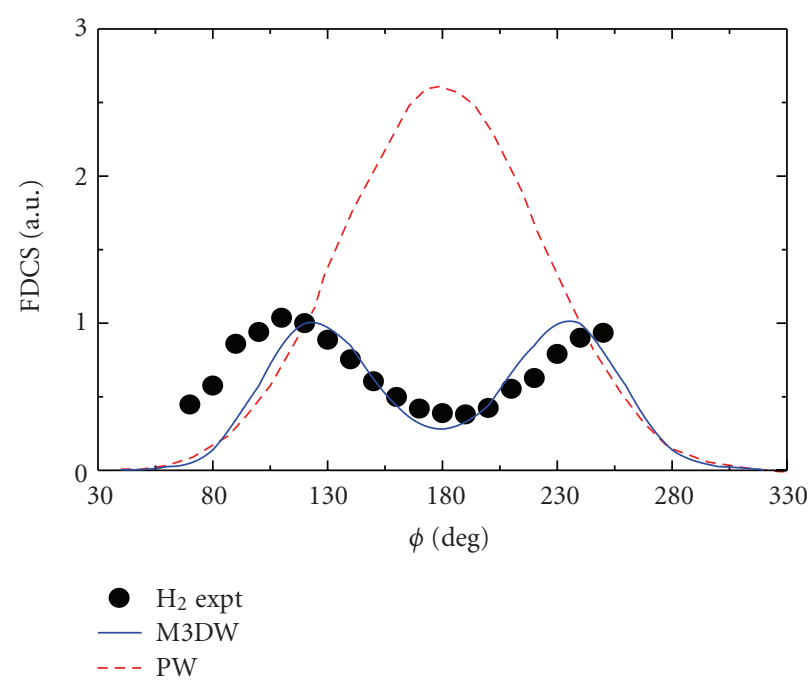

(a)

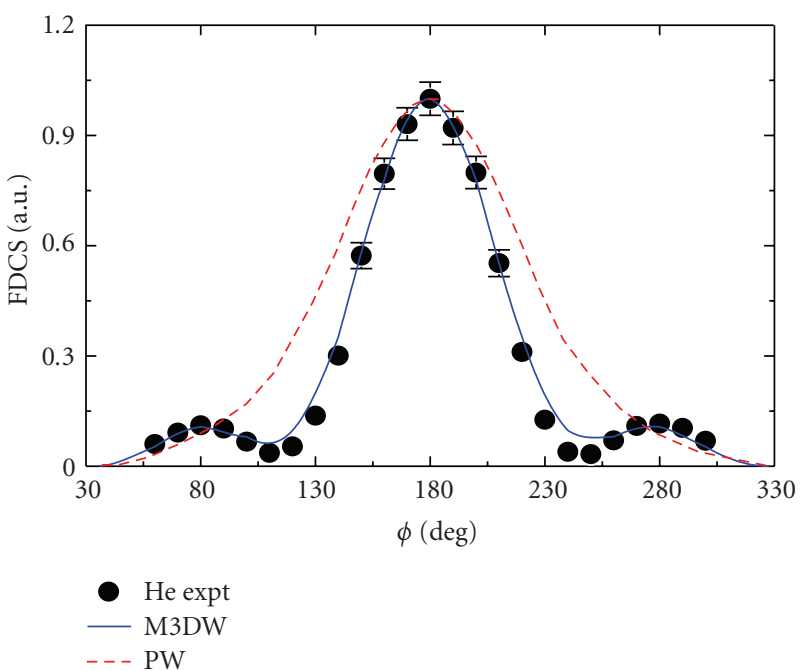

(b)

FIGURE 9: Experimental and theoretical FDCS for electron-impact ionization of $\mathrm{H}_{2}$ and $\mathrm{He}$ in the perpendicular plane. The outgoing electron energies were $E_{a}=E_{b}=10 \mathrm{eV}$ in both cases. The solid line is M3DW results and the dashed line is an equivalent calculation with the incident distorted-wave replaced by a plane wave.

change the nuclear part. Recall that the nuclear part consists of the charge of the nucleus distributed uniformly on a thin spherical shell centered on the center of mass. For $\mathrm{H}_{2}$, this means that we have a charge of +2 distributed on a thin spherical shell of radius $0.7 \mathrm{a}_{0}$. To investigate the effect of the nuclear charge distribution, we performed calculations with different sized radii decreasing to a point charge and the results are shown in Figure 10. It is seen that the $180^{\circ}$ minimum becomes a maximum for a point charge nucleus. We performed a similar calculation for $\mathrm{He}$ in which the nuclear charge was placed on a shell of increasing radius and all other aspects of the calculation remained unchanged and again the maximum became a minimum. 


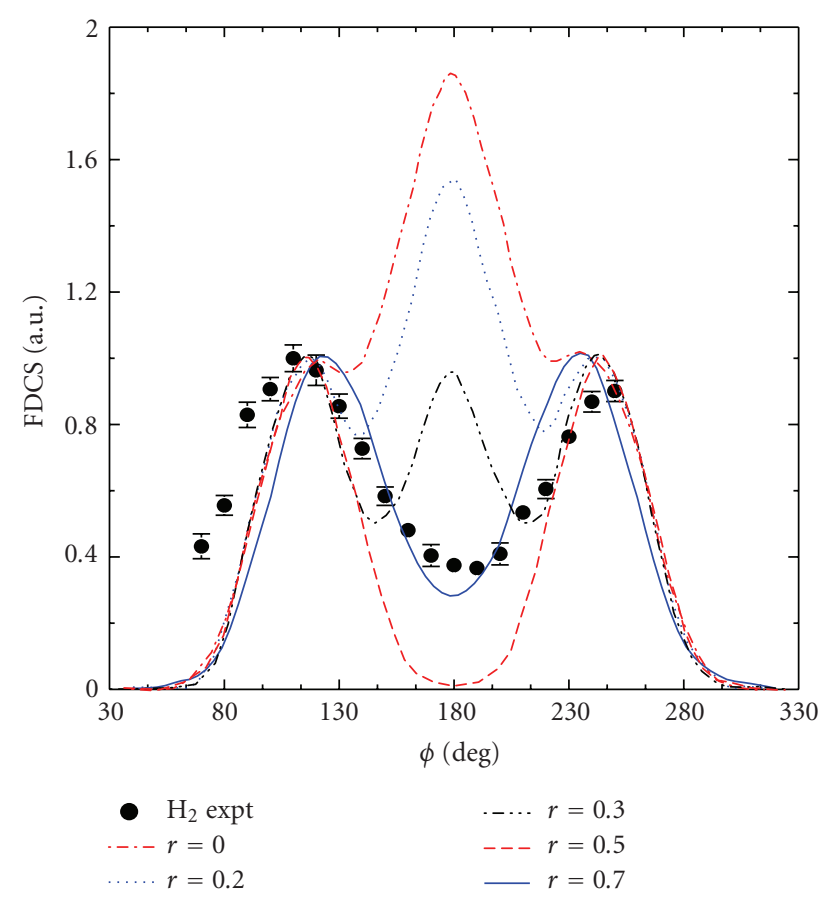

FIGURE 10: Experimental and theoretical FDCS for electron-impact ionization of $\mathrm{H}_{2}$ in the perpendicular plane. The outgoing electron energies were $E_{a}=E_{b}=10 \mathrm{eV}$ in both cases. The M3DW calculations are for different nuclear separations with $r=0.0,0.2,0.3,0.5$, and $0.7 \mathrm{a}_{0}$ as shown in the figure.

So, it is clear that the nuclei are responsible for the difference between the two targets but what is the physics behind this? If one performs a simple classical impact parameter calculation, it is seen that, for the kinematics of the experiment, an impact parameter of about $0.5 \mathrm{a}_{0}$ is required to scatter into the perpendicular plane. If the classical collision between the projectile electron and target electron took place inside a spherical shell of charge, there would be no attractive force on the electrons. On the other hand, if there were a point nuclei close by, there would be a strong attractive force. Consequently, these observations suggest the following collision mechanisms. For $\mathrm{He}$, elastic scattering places the projectile electron near the nuclei where it has a binary collision with the target electron which produces the $90^{\circ}$ and $270^{\circ}$ peaks. However, since both electrons are close to the target nucleus, there will be a strong attractive force which can also cause $180^{\circ}$ backscattering. For $\mathrm{H}_{2}$, we have the same binary collision but now the two electrons are inside a spherical shell so there is no attractive force which can cause backscattering so all we get are the $90^{\circ}$ and $270^{\circ}$ peaks. If this is the proper interpretation, then one would expect a $180^{\circ}$ maximum for molecules with a nucleus at the center of mass and this has been observed for $\mathrm{CO}_{2}$. However, we would also expect a $180^{\circ}$ maximum for $\mathrm{H}_{2} \mathrm{O}$ as mentioned above and the theory predicts the maximum but it was not found in the experiment.

\section{Conclusions}

Although significant theoretical progress for calculating FDCS for electron-impact ionization of molecules has been made in the last few years, there is still much to be done. While the experimental techniques are significantly ahead of the theoretical developments, this is an exciting time since experiments are able to produce excellent data with great detail which provides very stringent tests for theoretical models. The work that has been done so far has provided some valuable insights into the mechanisms of molecular ionization as well as provided some unanswered questions. For example, is the simple model of Al-Hagan et al. [110] correct which states that molecules which have a nucleus at the center of mass will have strong back-to-back scattering in the perpendicular plane while molecules which do not have a nucleus at the center of mass will have weak back-to-back scattering? We have one data set supporting this model and one data set that does not support it.

For the simplest molecule $\mathrm{H}_{2}$ and high incident energies, both the M3DW and FBA-TCC results are in qualitative agreement with the binary peak and poor agreement for the recoil peak. For low incident energies, both the M3DW and TDCC provide very good agreement with the shape of the data in the perpendicular plane. The TDCC gives good shape agreement and relative normalization for out-of-plane angles greater than $45^{\circ}$ and rather poor agreement for angles in and near the scattering plane. Surprisingly, the M3DW predicts the relative magnitudes of the cross-section for different planes better than the TDCC. This lack of agreement between experiment and theory for the smallest molecule is a major challenge that needs to be solved.

The larger diatomic molecule that has received the most attention recently is $\mathrm{N}_{2}$. For intermediate energies $(75 \mathrm{eV}$ and $150 \mathrm{eV}$ ), there is reasonable good agreement between experiment and theory for the binary peak and very bad agreement for the recoil peak. For higher incident energies, one would expect the theoretical approaches to be better but this is not the case. For higher energies, the M3DW and FBA-TCC binary peaks are in better agreement with each other than with experiment. The experimental location for the $2 \sigma_{g}$ and $\left(3 \sigma_{g}, 1 \pi_{u}, 2 \sigma_{u}\right)$ binary peaks are shifted to larger scattering angles as compared to theory. Although the FBATCC is in qualitative agreement with the recoil peak for ionization of the $\left(3 \sigma_{g}, 1 \pi_{u}, 2 \sigma_{u}\right)$ states, there is no similarity at all between theory and experiment for the $2 \sigma_{g}$ recoil peak.

For the case of low energy ionization of the $3 a_{1}$ state of $\mathrm{H}_{2} \mathrm{O}$, results completely opposite from $\mathrm{H}_{2}$ were found. For $\mathrm{H}_{2}$, the best agreement between experiment and theory was in the perpendicular plane. For $\mathrm{H}_{2} \mathrm{O}$, the best agreement between experiment and theory is in the scattering plane and the worst agreement is in the perpendicular plane. For $\mathrm{H}_{2}$, the largest cross-section was found for $\psi=45^{\circ}$ and for $\mathrm{H}_{2} \mathrm{O}$ the largest cross-section is in the scattering plane. Since the M3DW has been moderately successful for $\mathrm{N}_{2}$, the big question is whether or not it will also work for even larger molecules and more theoretical and experimental works are required to answer this question. 
Finally for the even larger molecule of FA, the fact that the M3DW produced reasonably good agreement with the high energy EMS measurements is very encouraging and indicates the validity of the OAMO at least for the $10 a^{\prime}$ state. Unfortunately, the experimental data could not resolve the $10 a^{\prime}$ and $2 a^{\prime \prime}$ states, and since the OAMO approximation is known to not be valid for the $2 a^{\prime \prime}$ state, no definite conclusions can be made until either we have an improved experimental resolution or a M3DW calculation that does not make the OAMO approximation.

There has been considerable interest in the possibility of two-center double-slit interference effects for scattering from diatomic molecules. Stia et al. [149] predicted that the crosssection for molecular hydrogen could be expressed as the cross-section for atomic hydrogen times an interference factor. Consequently, one could look for double-slit interference effects by comparing molecular and atomic cross-sections. Two experiments for $\mathrm{H}_{2}$ and one experiment for $\mathrm{N}_{2}$ have found evidence for interference using this method. However, this represents indirect evidence for interference at best and it would be much more satisfying to have a more direct method for observing a double slit interference effect.

Finally, the M3DW results presented so far all rely on the OAMO approximation which is potentially valid for a limited number of states and a limited range of scattering angles. Although the approximation has proved to be surprisingly successful for several cases, it is clearly highly desirable to develop a M3DW calculation that does not use this approximation.

\section{Acknowledgments}

This work was supported by the American NSF under Grant no. 0757749. Ola Al-Hagan would like to acknowledge the support of the Saudi Ministry of Higher Education and King Abdullah Bin Abdul-Aziz Scholarship.

\section{References}

[1] V. Mergel, R. Dörner, Kh. Khayyat, et al., "Strong correlations in the He ground state momentum wave function observed in the fully differential momentum distributions for the $p+$ He transfer ionization process," Physical Review Letters, vol. 86, no. 11, pp. 2257-2260, 2001.

[2] H. Schmidt-Böcking, V. Mergel, L. Schmidt, et al., "Dynamics of ionization processes studied with the COLTRIMS method-new insight into e-e correlation," Radiation Physics and Chemistry, vol. 68, no. 1-2, pp. 41-50, 2003.

[3] H. Schmidt-Böcking, V. Mergel, R. Dörner, et al., "Revealing the non- $s^{2}$ contributions in the momentum wave function of ground-state He," Europhysics Letters, vol. 62, no. 4, pp. 477483, 2003.

[4] H. Schmidt-Böcking, V. Mergel, R. Dörner, et al., "Experimental investigation of the asymptotic momentum wave function of He ground state," in Correlations, Polarization and Ionization in Atomic Systems, vol. 604 of AIP Conference Proceedings, p. 120, 2001.

[5] M. Schulz, R. Moshammer, D. Fischer, et al., "Threedimensional imaging of atomic four-body processes," Nature, vol. 422, no. 6927, pp. 48-50, 2003.
[6] T. N. Rescigno, M. Baertschy, W. A. Isaacs, and C. W. McCurdy, "Collisional breakup in a quantum system of three charged particles," Science, vol. 286, no. 5449, pp. 2474-2479, 1999.

[7] I. Bray, "Low-energy electron-impact ionization of atomic hydrogen with equal energy outgoing electrons," Journal of Physics B, vol. 33, no. 3, pp. 581-595, 2000.

[8] J. Colgan and M. S. Pindzola, "Double- and triple-differential cross sections for the low-energy electron-impact ionization of hydrogen," Physical Review A, vol. 74, no. 1, Article ID 012713, 2006.

[9] K. Bartschat and I. Bray, "Electron-impact ionization of atomic hydrogen from the $1 \mathrm{~S}$ and $2 \mathrm{~S}$ states," Journal of Physics $B$, vol. 29, no. 15, pp. L577-L583, 1996.

[10] I. Bray, "Close-coupling approach to coulomb three-body problems," Physical Review Letters, vol. 89, no. 27, Article ID 273201, 4 pages, 2002.

[11] I. Bray, D. V. Fursa, A. S. Kheifets, and A. T. Stelbovics, "Electrons and photons colliding with atoms: development and application of the convergent close-coupling method," Journal of Physics B, vol. 35, no. 15, pp. R117-R146, 2002.

[12] J. Colgan, M. S. Pindzola, G. Childers, and M. A. Khakoo, "Low-energy electron-impact single ionization of helium," Physical Review A, vol. 73, no. 4, Article ID 042710, 7 pages, 2006.

[13] D. H. Madison, R. V. Calhoun, and W. N. Shelton, "Tripledifferential cross sections for electron-impact ionization of helium," Physical Review A, vol. 16, no. 2, pp. 552-562, 1977.

[14] C. T. Whelan and H. R. J. Walters, "A new version of the distorted-wave impulse approximation-application to coplanar symmetric ionization," Journal of Physics B, vol. 23, no. 17, pp. 2989-2995, 1990.

[15] X. Zhang, C. T. Whelan, and H. R. J. Walters, “(e,2e) cross sections for ionization of helium in coplanar symmetric geometry," Journal of Physics B, vol. 23, no. 17, pp. L509L516, 1990.

[16] X. Zhang, C. T. Whelan, and H. R. J. Walters, "Energy sharing $(\mathrm{e}, 2 \mathrm{e})$ collisions-ionisation of helium in the perpendicular plane," Journal of Physics B, vol. 23, no. 10, pp. L173-L178, 1990.

[17] E. P. Curran, C. T. Whelan, and H. R. J. Walters, "On the electron impact ionisation of $\mathrm{H}(1 \mathrm{~s})$ in coplanar asymmetric geometry," Journal of Physics B, vol. 24, pp. L19-L25, 1991.

[18] D. H. Madison, K. Bartschat, and J. L. Peacher, "Effect of nonspherical distorting potentials in a first-order distortedwave calculation," Physical Review A, vol. 44, no. 1, pp. 304309, 1991.

[19] X. Zhang, C. T. Whelan, and H. R. J. Walters, "Electron impact ionization of the noble gases in coplanar symmetric geometry over the energy range 100 to $1000 \mathrm{eV}$," Zeitschrift für Physik D, vol. 18, no. 4, pp. 309-310, 1991.

[20] L. Avaldi, R. Camilloni, P. Letardi, et al., "Absolute triple differential cross sections for helium at $300 \mathrm{eV}$," Zeitschrift für Physik D, vol. 23, no. 4, pp. 341-345, 1992.

[21] M. Chérid, F. Gélébart, A. Pochat, et al., "Absolute triple differential cross-section measurements in energy sharing kinematics and a coplanar asymmetric geometry at 100 and $200 \mathrm{eV}$ on He," Zeitschrift für Physik D, vol. 23, no. 4, pp. 347351, 1992.

[22] T. Rösel, J. Röder, L. Frost, et al., "Absolute triple differential cross section for ionization of helium near threshold," Physical Review A, vol. 46, no. 5, pp. 2539-2552, 1992. 
[23] S. Jones, D. H. Madison, and M. K. Srivastava, "Nearthreshold ionization of hydrogen and helium by electron impact," Journal of Physics B, vol. 25, pp. 1899-1914, 1992.

[24] C. Pan and A. F. Starace, "Angular distributions for nearthreshold $(\mathrm{e}, 2 \mathrm{e})$ processes for $\mathrm{H}, \mathrm{He}$, and other rare-gas targets," Physical Review A, vol. 45, no. 7, pp. 4588-4603, 1992.

[25] X. Zhang, C. T. Whelan, and H. R. J. Walters, "Distortedwave Born approximation calculations of (e, 2e) reactions," Zeitschrift für Physik D, vol. 23, no. 4, pp. 301-308, 1992.

[26] X. Zhang, C. T. Whelan, H. R. J. Walters, et al., “(e, 2e) cross sections for inner-shell ionization of argon and neon," Journal of Physics B, vol. 25, pp. 4325-4335, 1992.

[27] H. R. J. Walters, H. Ast, C. T. Whelan, et al., "Relativistic (e, 2e) collisions on atomic inner shells in symmetric geometry," Zeitschrift für Physik D, vol. 23, no. 4, pp. 353-357, 1992.

[28] L. Avaldi, R. Camilloni, R. Multari, et al., "Coplanar asymmetric (e,2e) experiments on xenon $4 \mathrm{~d}$ and $5 \mathrm{p}$ orbitals," Physical Review A, vol. 48, no. 2, pp. 1195-1203, 1993.

[29] S. Jones, D. H. Madison, A. Franz, and P. L. Altick, “Threebody distorted-wave Born approximation for electron-atom ionization," Physical Review A, vol. 48, no. 1, pp. R22-R25, 1993.

[30] A. Pochat, X. Zhang, C. T. Whelan, et al., "Absolute lowenergy experimental cross sections for $(\mathrm{e}, 2 \mathrm{e})$ processes on helium," Physical Review A, vol. 47, no. 5, pp. R3483-R3486, 1993.

[31] C. T. Whelan, R. J. Allan, and H. R. J. Walters, "PCI, polarization and exchange effects in (e,2e) collisions," Journal De Physique IV, vol. 3, no. 6, pp. 39-49, 1993.

[32] C. T. Whelan, R. J. Allan, H. R. J. Walters, and X. Zhang, " $(\mathrm{e}, 2 \mathrm{e})$, effective charges, distorted waves an all that!," in $(e, 2 e)$ and Related Processes, C. T. Whelan, H. R. J. Walters, A. Lahmam-Bennani, and H. Ehrhardt, Eds., pp. 1-32, Kluwer Academic Publishers, Dordrecht, The Netherland, 1993.

[33] H. Ast, S. Keller, C. T. Whelan, H. R. J. Walters, and R. M. Dreizler, "Electron-impact ionization of the K shell of silver and gold in coplanar asymmetric geometry," Physical Review A, vol. 50, no. 1, pp. R1-R3, 1994.

[34] S. Jones and D. H. Madison, "Asymptotically-correct distorted-wave calculations for low-energy electron-impact ionization of helium," Journal of Physics B, vol. 27, pp. 14231428, 1994.

[35] S. Keller, C. T. Whelan, H. Ast, H. R. J. Walters, and R. M. Dreizler, "Relativistic distorted-wave Born calculations for $(\mathrm{e}, 2 \mathrm{e})$ processes on inner shells of heavy atoms," Physical Review A, vol. 50, no. 5, pp. 3865-3877, 1994.

[36] C. T. Whelan, R. J. Allan, J. Rasch, et al., "Coulomb threebody effects in $(\mathrm{e}, 2 \mathrm{e})$ collisions: the ionization of $\mathrm{H}$ in coplanar symmetric geometry," Physical Review A, vol. 50, no. 5, pp. 4394-4396, 1994.

[37] H. Ast, S. Keller, R. M. Dreizler, C. T. Whelan, L. U. Ancarani, and H. R. J. Walters, "On the position of the binary peak in relativistic (e, 2e) collisions," Journal of Physics B, vol. 29, no. 15, pp. L585-L590, 1996.

[38] B. Granitza, A. X. Guo, J. M. Hurn, et al., "Spin effects in the (e, 2e) cross section of xenon," Australian Journal of Physics, vol. 49, no. 2, pp. 383-401, 1996.

[39] X. Guo, J. M. Hurn, J. Lower, et al., "Fine structure effect in electron impact ionization," Physical Review Letters, vol. 76, no. 8, pp. 1228-1231, 1996.
[40] S. Keller, R. M. Dreizler, L. U. Ancarani, H. R. J. Walters, H. Ast, and C. T. Whelan, "Theoretical analysis of the relativistic first order Born approximation for inner shell (e, 2e) processes," Zeitschrift für Physik D, vol. 37, no. 3, pp. 191196, 1996.

[41] S. Keller, R. M. Dreizler, H. Ast, C. T. Whelan, and H. R. J. Walters, "Theory of $(\mathrm{e}, 2 \mathrm{e})$ processes with spin-polarized relativistic electrons," Physical Review A, vol. 53, no. 4, pp. 2295-2302, 1996.

[42] D. H. Madison, V. D. Kravtsov, S. Jones, and R. P. McEachran, "Ionization of heavy inert gases by spin-polarized electrons," Canadian Journal of Physics, vol. 74, no. 11-12, pp. 816-821, 1996.

[43] D. H. Madison, V. D. Kravtsov, S. Jones, and R. P. McEachran, "Fine-structure effect for (e,2e) collisions," Physical Review A, vol. 53, no. 4, pp. 2399-2406, 1996.

[44] J. Röder, J. Rasch, K. Jung, et al., "Coulomb three-body effects in low-energy impact ionization of $\mathrm{H}(1 \mathrm{~s})$, , Physical Review A, vol. 53, no. 1, pp. 225-233, 1996.

[45] C. T. Whelan, H. Ast, H. R. J. Walters, S. Keller, and R. M. Dreizler, "Relativistic-energy-sharing $(\mathrm{e}, 2 \mathrm{e})$ collisions in coplanar constant $\theta 1,2$ geometry," Physical Review A, vol. 53, no. 5, pp. 3262-3270, 1996.

[46] C. T. Whelan, H. R. J. Walters, S. Keller, H. Ast, J. Rasch, and R. M. Dreizler, "Inner shell (e, 2e) processes," Canadian Journal of Physics, vol. 74, no. 11-12, pp. 804-810, 1996.

[47] A. Dorn, A. Elliott, X. Guo, et al., “(e, 2e) collisions on xenon with spin-polarized electrons," Journal of Physics B, vol. 30, no. 18, pp. 4097-4121, 1997.

[48] R. M. Dreizler, H. Ast, S. Keller, C. T. Whelan, L. U. Ancarani, and H. R. J. Walters, "Structure of the triply differential cross section in the recoil region in relativistic electron-impact ionization: II. Theoretical analysis," Journal of Physics B, vol. 30, no. 2, pp. L77-L80, 1997.

[49] J. Rasch, C. T. Whelan, R. J. Allan, S. P. Lucey, and H. R. J. Walters, "Strong interference effects in the triple differential cross section of neutral-atom targets," Physical Review A, vol. 56, no. 2, pp. 1379-1383, 1997.

[50] J. Rasch, M. Zitnik, L. Avaldi, et al., "Theoretical and experimental investigation of the triple-differential cross sections for electron-impact ionization of $\operatorname{Kr}(4 \mathrm{p})$ and $\mathrm{Xe}(\mathrm{Sp})$ at 1-keV impact energy," Physical Review A, vol. 56, no. 6, pp. 4644-4655, 1997.

[51] S. Rioual, B. Rouvellou, A. Pochat, et al., "Triple differential cross sections for the electron-impact ionization of argon and neon," Journal of Physics B, vol. 30, no. 13, pp. L475-L480, 1997.

[52] L. U. Ancarani, S. Keller, H. Ast, C. T. Whelan, H. R. J. Walters, and R. M. Dreizler, "Influence of Coulomb boundary conditions for (e, 2e) processes on the K-shell of high-Z atoms," Journal of Physics B, vol. 31, no. 3, pp. 609623, 1998.

[53] S. Keller, H. Ast, L. U. Ancarani, C. T. Whelan, H. R. J. Walters, and R. M. Dreizler, "Remark on the theory of threebody effects in relativistic (e, 2e) processes," Journal of Physics B, vol. 31, no. 20, pp. L873-L876, 1998.

[54] D. H. Madison, V. D. Kravtsov, and S. Mazevet, "Role of exchange scattering in spin-dependent (e,2e) collisions," Journal of Physics B, vol. 31, no. 1, pp. L17-L25, 1998.

[55] S. Mazevet, I. E. McCarthy, and E. Weigold, "Parametrization of a spin-polarized (e ,2e) experiment," Physical Review A, vol. 57, no. 3, pp. 1881-1891, 1998. 
[56] S. Mazevet, I. E. McCarthy, D. H. Madison, and E. Weigold, "Semirelativistic DWBA for the ionization of closed shell atoms at intermediate energies," Journal of Physics B, vol. 31, no. 10, pp. 2187-2202, 1998.

[57] C. Mette, T. Simon, C. Herting, G. F. Hanne, and D. H. Madison, "Spin-resolved triple differential cross sections of xenon," Journal of Physics B, vol. 31, no. 20, pp. 4689-4700, 1998.

[58] O. Rath Spivack, J. Rasch, C. T. Whelan, R. J. Allan, and H. R. J. Walters, "(e, 2e) processes with positive-ion targets in coplanar symmetric geometry," Journal of Physics B, vol. 31, no. 4, pp. 845-858, 1998.

[59] B. Rouvellou, S. Rioual, J. Röder, et al., "Coulomb threebody effects in electron-impact ionization of argon," Physical Review A, vol. 57, no. 5, pp. 3621-3626, 1998.

[60] M. Streun, G. Baum, W. Blask, et al., "Spin dependence of (e, 2e) collisions on lithium at $54.4 \mathrm{eV}$," Journal of Physics B, vol. 31, no. 19, pp. 4401-4411, 1998.

[61] L. U. Ancarani, H. Ast, S. Keller, R. M. Dreizler, C. T. Whelan, and H. R. J. Walters, "Analysis of semi-relativistic approximations for relativistic (e,2e) experiments," Journal De Physique IV, vol. 9, no. 6, pp. Pr6-225-Pr6-228, 1999.

[62] S. J. Cavanagh, B. Lohmann, J. Rasch, C. T. Whelan, and H. R. J. Walters, "Experimental and theoretical determination of the triple differential cross section for $\operatorname{Kr}(4 \mathrm{p})$ electronimpact ionization," Physical Review A, vol. 60, no. 4, pp. 2977-2982, 1999.

[63] S. Keller, L. U. Ancarani, H. Ast, R. M. Dreizler, H. R. J. Walters, and C. T. Whelan, "Analysis of relativistic (e,2e) experiments in coplanar asymmetric geometry," Journal De Physique IV, vol. 9, no. 6, pp. Pr6-215-Pr6-218, 1999.

[64] S. Keller, R. M. Dreizler, L. U. Ancarani, H. Ast, H. R. J. Walters, and C. T. Whelan, "Interpretation of relativistic $(\mathrm{e}, 2 \mathrm{e})$ experiments in coplanar asymmetric geometry: atomic-number-dependent effects," Physical Review A, vol. 59, no. 2-3, pp. 1284-1290, 1999.

[65] Y. Khajuria and D. N. Tripathi, "Geometry effects on the (e,2e) triple differential cross sections of $\mathrm{Li}^{+}, \mathrm{Na}^{+}$, and $\mathrm{K}^{+}$," Physical Review A, vol. 59, no. 2-3, pp. 1197-1207, 1999.

[66] D. H. Madison, S. Jones, and A. Humberston, "Role of electron-electron correlation in (e,2e) reactions," Journal De Physique IV, vol. 9, no. 6, pp. Pr6-31-Pr6-34, 1999.

[67] I. Taouil, A. Duguet, A. Lahmam-Bennani, et al., “(e, 2e) ionization of the $2 \mathrm{p}$ orbital of argon," Journal of Physics B, vol. 32, no. 1, pp. L5-L11, 1999.

[68] D. A. Biava, H. P. Saha, E. Engel, et al., "Exchange effects in low energy electron impact ionization of the inner and outer shells of argon," Journal of Physics B, vol. 35, no. 2, pp. 293307, 2002.

[69] D. A. Biava, K. Bartschat, H. P. Saha, and D. H. Madison, "Accuracy of local exchange in the calculation of continuum wavefunctions," Journal of Physics B, vol. 35, no. 24, pp. 51215130, 2002.

[70] R. I. Campeanu, R. P. McEachran, and A. D. Stauffer, "Distorted-wave models in positron impact ionization of atoms," Nuclear Instruments and Methods in Physics Research, Section B, vol. 192, no. 1-2, pp. 146-149, 2002.

[71] M. Kampp, P. J. P. Roche, C. T. Whelan, D. H. Madison, J. Rasch, and H. R. J. Walters, "The electron impact ionization of magnesium (2p)," Journal of Physics B, vol. 35, no. 10, pp. 2325-2336, 2002.
[72] Y. Khajuria, L. Q. Chen, X. J. Chen, and K. Z. Xu, "The triple differential cross sections of ionic targets in the coplanar to perpendicular plane geometry," Journal of Physics B, vol. 35, no. 1, pp. 93-110, 2002.

[73] Y. Khajuria, L. Q. Chen, X. J. Chen, and K. Z. Xu, “(e,2e) triple-differential cross sections of helium and argon at 64.6 eV," Physical Review A, vol. 65, no. 4, Article ID 042706, 6 pages, 2002.

[74] L. Q. Chen, Y. Khajuria, X. J. Chen, and K. Z. Xu, "Triple differential cross-sections of $\mathrm{Ne}\left(2 \mathrm{~S}^{2}\right)$ in coplanar to perpendicular plane geometry," European Physical Journal D, vol. 26, no. 2, pp. 141-146, 2003.

[75] A. Prideaux and D. H. Madison, "Role of the postcollision interaction in electron-impact ionization of argon and krypton," Physical Review A, vol. 67, no. 5, Article ID 052710, 8 pages, 2003.

[76] G. Purohit, A. S. Bhullar, and K. K. Sud, "(e, 2e) Triple differential cross sections of $\mathrm{He}, \mathrm{Ne}, \mathrm{Ar}, \mathrm{Kr}$ and $\mathrm{Xe}$ atoms in coplanar to perpendicular plane geometry," Indian Journal of Physics B, vol. 77, no. 2, pp. 177-184, 2003.

[77] M. Kampp, S. Kawano, P. J. P. Roche, et al., "On the observation of the fine structure effect in non-relativistic (e, 2e) processes," European Physical Journal D, vol. 29, no. 1, pp. 17-19, 2004.

[78] A. Prideaux and D. H. Madison, "Doubly differential cross sections for ionization of xenon by spin-polarized electrons," Journal of Physics B, vol. 37, no. 22, pp. 4423-4433, 2004.

[79] G. Purohit, R. Choubisa, V. Patidar, and K. K. Sud, "Spin asymmetry in (e, 2e) processes on $\mathrm{Li}, \mathrm{Be}^{+}, \mathrm{B}^{+2}$ and $\mathrm{C}^{+3}$ targets by transversely polarized electrons," Physica Scripta, vol. 69, no. 3, pp. 208-215, 2004.

[80] R. K. Chauhan, M. K. Srivastava, and R. Srivastava, "Triple differential cross sections of coplanar symmetric $(e, 2 e)$ processes on calcium at low energies," Physical Review A, vol. 71, no. 3, Article ID 032708, 5 pages, 2005.

[81] M. Stevenson, G. J. Leighton, A. Crowe, K. Bartschat, O. K. Vorov, and D. H. Madison, "Experimental and theoretical (e, 2e) studies of argon (3p) ionization in asymmetric geometry," Journal of Physics B, vol. 38, no. 4, pp. 433-440, 2005.

[82] F. Catoire, E. M. Staicu-Casagrande, M. Nekkab, C. Dal Cappello, K. Bartschat, and A. Lahmam-Bennani, "Investigation of the (e, 2e) single ionization of $\mathrm{He}$ and Ar at large energy loss close to minimum momentum transfer," Journal of Physics B, vol. 39, no. 12, pp. 2827-2838, 2006.

[83] M. K. Srivastava, R. K. Chauhan, and R. Srivastava, "Coplanar doubly symmetric (e,2e) process on sodium and potassium," Physical Review A, vol. 74, no. 6, Article ID 064701, 2006.

[84] K. K. Sud, G. Purohit, and A. S. Bhullar, "Electron dichroism effects in the relativistic (e, 2e) process for K-shell ionization of atoms," Pramana, vol. 62, no. 5, pp. 1157-1166, 2004.

[85] Y. Khajuria and P. C. Deshmukh, "Xe(4d) triple differential cross section: modified semiclassical exchange approximation in electron-atom collision," Journal of Physics B, vol. 39, no. 3, pp. 569-576, 2006.

[86] R. Panajotovic, J. Lower, E. Weigold, A. Prideaux, and D. H. Madison, "(e,2e) measurements on xenon: reexamination of the fine-structure effect," Physical Review A, vol. 73, no. 5, Article ID 052701, 2006.

[87] Y. Khajuria, S. Sunil Kumar, and P. C. Deshmukh, “(e,2e) triple differential cross section of $\mathrm{Mg}$ in coplanar symmetric geometry," Physical Review A, vol. 75, no. 2, Article ID 022708, 2007. 
[88] S. Sunil Kumar, Y. Khajuria, and P. C. Deshmukh, “(e, 2e) study of Ca in coplanar symmetric geometry at low impact energies using distorted wave born approximation," Journal of Physics: Conference Series, vol. 80, no. 1, Article ID 012021, 2007.

[89] E. M. Staicu Casagrande, F. Catoire, A. Naja, et al., "New coplanar $(\mathrm{e}, 2 \mathrm{e})$ experiments for the ionisation of $\mathrm{He}$ and Ar atoms," Journal of Electron Spectroscopy and Related Phenomena, vol. 161, no. 1-3, pp. 27-30, 2007.

[90] S. Bellm, J. Lower, R. P. McEachran, E. Weigold, C. RyanAnderson, and D. H. Madison, "Spin- and fine-structureresolved ionization of krypton," Physical Review A, vol. 78, no. 6, Article ID 062707, 2008.

[91] P. Bolognesi, L. Pravica, S. Veronesi, et al., "Mg 2p ionization by electron impact," Physical Review A, vol. 77, no. 5, Article ID 054704, 2008.

[92] R. Dey, A. C. Roy, and C. Dal Cappello, "Electron impact single ionization of helium with large energy transfer," Nuclear Instruments and Methods in Physics Research, Section B, vol. 266, no. 2, pp. 242-249, 2008.

[93] U. Hitawala, G. Purohit, and K. K. Sud, "(e, 2e) triple differential cross sections of alkali and alkali earth atoms: $\mathrm{Na}$, $\mathrm{K}$ and Mg, Ca," Journal of Physics B, vol. 41, no. 3, Article ID 035205, 2008.

[94] Y. Khajuria and P. C. Deshmukh, “(e,2e) study of Ca in coplanar symmetric geometry," Physical Review A, vol. 78, no. 2, Article ID 024702, 2008.

[95] A. S. Kheifets, A. Naja, E. M. S. Casagrande, and A. LahmamBennani, "DWBA-G calculations of electron impact ionization of noble gas atoms," Journal of Physics B, vol. 41, no. 14, Article ID 145201, 2008.

[96] A. S. Kheifets, A. Naja, E. M. S. Casagrande, and A. LahmamBennani, "DWBA-G calculations of electron impact ionization of noble gas atoms," Journal of Physics B, vol. 41, no. 20, Article ID 209801, 2008.

[97] J. M. Martinez, H. R. J. Walters, and C. T. Whelan, "The electron impact ionization of one- and two-electron atoms and ions close to the ionization threshold," Journal of Physics B, vol. 41, no. 6, Article ID 065202, 2008.

[98] A. Naja, E. M. S. Casagrande, A. Lahmam-Bennani, et al., “(e, 2e) triple differential cross-sections for ionization beyond helium: the neon case at large energy transfer," Journal of Physics B, vol. 41, Article ID 085205, 8 pages, 2008.

[99] Y. Khajuria, S. Sunil Kumar, and P. C. Deshmukh, "Triple differential cross section in $(e, 2 e)$ collisions for atomic potassium," Physics Letters A, vol. 373, no. 48, pp. 4442-4446, 2009.

[100] M. A. Stevenson, L. R. Hargreaves, B Lohmann, et al., "Fully differential cross-section measurements for electron-impact ionization of neon and xenon," Physical Review A, vol. 79, no. 1, Article ID 012709, 8 pages, 2009.

[101] M. Brauner, J. S. Briggs, and H. Klar, "Triply-differential cross sections for ionisation of hydrogen atoms by electrons and positrons," Journal of Physics B, vol. 22, no. 14, pp. 22652287, 1989.

[102] P. J. Redmond, unpublished.

[103] L. Rosenberg, "Variational methods in charged-particle collision theory," Physical Review D, vol. 8, no. 6, pp. 18331843, 1973.

[104] E. O. Alt and A. M. Mukhamedzhanov, "Asymptotic solution of the Schrödinger equation for three charged particles," Physical Review A, vol. 47, no. 3, pp. 2004-2022, 1993.
[105] J. Berakdar, "Approximate analytical solution of the quantum-mechanical three-body Coulomb continuum problem," Physical Review A, vol. 53, no. 4, pp. 2314-2326, 1996.

[106] A. M. Mukhamedzhanov and M. Lieber, "Asymptotic wave function for three charged particles in the continuum," Physical Review A, vol. 54, no. 4, pp. 3078-3085, 1996.

[107] Y. Qiu, J.-Z. Tang, J. Burgdörfer, and J. Wang, "Double photoionization of helium from threshold to high energies," Physical Review A, vol. 57, no. 3, pp. R1489-R1492, 1998.

[108] J. Botero and J. H. Macek, "Threshold angular distributions of (e, 2e) cross sections of helium atoms," Physical Review Letters, vol. 68, no. 5, pp. 576-579, 1992.

[109] S. J. Ward and J. H. Macek, "Wave functions for continuum states of charged fragments," Physical Review A, vol. 49, no. 2, pp. 1049-1056, 1994.

[110] O. Al-Hagan, C. Kaiser, D. Madison, and A. J. Murray, "Atomic and molecular signatures for charged-particle ionization," Nature Physics, vol. 5, no. 1, pp. 59-63, 2009.

[111] D. S. F. Crothers and J. F. McCann, "Ionisation of atoms by ion impact," Journal of Physics B, vol. 16, no. 17, pp. 32293242, 1983.

[112] S. Jones and D. H. Madison, "Evidence of initial-state twocenter effects for (e, 2e) reactions," Physical Review Letters, vol. 81, no. 14, pp. 2886-2889, 1998.

[113] S. Jones and D. H. Madison, "Ionization of hydrogen atoms by fast electrons," Physical Review A, vol. 62, no. 4, Article ID 042701, 10 pages, 2000.

[114] D. Madison, M. Schulz, S. Jones, M. Foster, R. Moshammer, and J. Ullrich, "Comparison of theoretical and absolute experimental fully differential cross sections for ion-atom impact ionization," Journal of Physics B, vol. 35, no. 15, pp. 3297-3314, 2002.

[115] D. H. Madison, D. Fischer, M. Foster, et al., "Probing scattering wave functions close to the nucleus," Physical Review Letters, vol. 91, no. 25, Article ID 253201, pp. 1-4, 2003.

[116] M. Foster, D. H. Madison, J. L. Peacher, et al., "Fully differential cross sections for $\mathrm{C}^{6+}$ single ionization of helium," Journal of Physics B, vol. 37, no. 8, pp. 1565-1580, 2004.

[117] A. Salin, "Helium ionisation by high-energy ions at a function of impact parameter and projectile scattering angle," Journal of Physics B, vol. 22, no. 23, pp. 3901-3914, 1989.

[118] H. Fukuda, I. Shimamura, L. Vegh, and T. Watanabe, "Proton angular distributions and highly differential cross sections for ionization of helium by proton impact," Physical Review A, vol. 44, no. 3, pp. 1565-1576, 1991.

[119] H. Fukuda, T. Watanabe, I. Shimamura, and L. Vegh, "Energy and angular distributions of electrons emitted and ions recoiled in proton-impact ionization of helium atoms," Nuclear Instruments and Methods in Physics Research B, vol. 53, no. 4, pp. 410-415, 1991.

[120] X. Fang and J. F. Reading, "Projectile angular dependence of the ratio of double to single ionization of helium by fast proton impact," Nuclear Instruments and Methods in Physics Research B, vol. 53, no. 4, pp. 453-471, 1991.

[121] V. D. Rodriguez and R. O. Barrachina, "Projectile angular distribution in He single ionization by proton impact as a function of the ejected-electron energy," Physical Review A, vol. 57, no. 1, pp. 215-220, 1998.

[122] R. Moshammer, A. Perumal, M. Schulz, et al., "Three-body Coulomb problem probed by mapping the Bethe surface in ionizing ion-atom collisions," Physical Review Letters, vol. 87, no. 22, Article ID 223201, pp. 1-4, 2001. 
[123] J. Fiol, V. D. Rodrıguez, and R. O. Barrachina, "Electron capture to the continuum by proton and positron impact," Journal of Physics B, vol. 34, no. 5, pp. 933-944, 2001.

[124] M. Schulz, R. Moshammer, A. N. Perumal, and J. Ullrich, "Triply differential single-ionization cross sections in fast ion-atom collisions at large perturbation," Journal of Physics B, vol. 35, no. 7, pp. L161-L166, 2002.

[125] L. Gulyas, P. D. Fainstein, and A. Salin, "CDW-EIS theory of ionization by ion impact with Hartree-Fock description of the target," Journal of Physics B, vol. 28, pp. 245-257, 1995.

[126] J. Rasch, C. T. Whelan, S. P. Lucey, C. Dal Cappello, and H. R. J. Walters, "6-dimensional integrals and supercomputers," Computer Physics Communications, vol. 114, no. 1-3, pp. 378-384, 1998.

[127] F. W. Byron Jr., C. J. Joachain, and B. Piraux, "Eikonal-Born series theory of $(\mathrm{e}, 2 \mathrm{e})$ reactions in atomic hydrogen," Journal of Physics B, vol. 18, no. 15, pp. 3203-3218, 1985.

[128] P. J. Marchalant, J. Rasch, C. T. Whelan, D. H. Madison, and H. R. J. Walters, "First and second Born calculations of (e, 2e) excitation-ionization of helium," Journal of Physics B, vol. 32, no. 24, pp. L705-L710, 1999.

[129] P. J. Marchalant, B. Rouvellou, J. Rasch, et al., "Excitationionization of helium to $\mathrm{He}^{+}(n=2)$ at $365.8 \mathrm{eV}$," Journal of Physics B, vol. 33, no. 20, pp. L749-L754, 2000.

[130] P. J. Marchalant, C. T. Whelan, and H. R. J. Walters, "Secondorder effects in (e, 2e) excitation-ionization of helium to $\mathrm{He}^{+}(n=2)$," Journal of Physics B, vol. 31, no. 6, pp. 11411178, 1998.

[131] R. H. G. Reid, K. Bartschat, and A. Raeker, "Initialstate, final-state and higher-order effects in electron impact ionization of helium atoms," Journal of Physics B, vol. 31, no. 3, pp. 563-571, 1998.

[132] R. H. G. Reid, K. Bartschat, and A. Raeker, "Corrigendum: initial-state, final-state and higher-order effects in electron impact ionization of helium atoms," Journal of Physics B, vol. 33, pp. 5261-5262, 2000.

[133] Y. Fang and K. Bartschat, "Convergent second-order calculations for simultaneous electron-impact ionization-excitation of helium," Journal of Physics B, vol. 34, no. 2, pp. L19-L25, 2001.

[134] K. Bartschat and O. Vorov, "Channel-coupling, targetstructure, and second-order effects in electron-impact ionization of $\operatorname{Ar}(3 p)$ and $\operatorname{Ar}(3 s)$ ), Physical Review A, vol. 72, no. 2, Article ID 022728, 7 pages, 2005.

[135] Z. Chen, D. H. Madison, C. T. Whelan, and H. R. J. Walters, "Second-order distorted wave calculation for electron impact ionization of hydrogen," Journal of Physics B, vol. 37, no. 5, pp. 981-995, 2004.

[136] Z. Chen and D. H. Madison, "Second-order distorted wave calculation for electron-impact ionization of helium to $\mathrm{He}^{+}$ ( $n=1$ and 2 )," Journal of Physics B, vol. 38, no. 23, pp. 41954209, 2005.

[137] Z. Chen, D. H. Madison, and K. Bartschat, "Investigation of the closure and simplified Green's function approximations in second-order distorted-wave calculations for the (e, 2e) processes," Journal of Physics B, vol. 40, no. 12, pp. 23332344, 2007.

[138] E. Weigold and I. E. McCarthy, Electron Momentum Spectroscopy, Kluwer Academic/Plenum Publishers, New York, NY, USA, 1999.

[139] A. Lahmam-Bennani, I. Taouil, A. Duguet, M. Lecas, L. Avaldi, and J. Berakdar, "Origin of dips and peaks in the absolute fully resolved cross sections for the electron-impact double ionization of He," Physical Review A, vol. 59, no. 5, pp. 3548-3555, 1999.

[140] P. Week, B. Joulakian, and P. A. Hervieux, "Fivefold differential cross section of fast (e, 2e) ionization of $\mathrm{H}_{2}, \mathrm{D}_{2}$, and $\mathrm{T}_{2}$ by a Franck-Condon approach," Physical Review A, vol. 60, no. 4, pp. 3013-3019, 1999.

[141] P. Weck, O. A. Fojón, J. Hanssen, B. Joulakian, and R. D. Rivarola, "Two-effective center approximation for the single ionization of molecular hydrogen by fast electron impact," Physical Review A, vol. 63, no. 4, Article ID 042709, 6 pages, 2001.

[142] C. Champion, J. Hanssen, and P. A. Hervieux, "Influence of molecular orientation on the multiple differential cross sections for the (e, 2e) process on a water molecule," Physical Review A, vol. 63, no. 5, Article ID 052720, 9 pages, 2001.

[143] F. Elboudali and B. Joulakian, "Two-centre partial-wave calculations for the multiply differential cross section of the simple ionization of diatomic lithium $\mathrm{Li}_{2}$ by fast electron impact," Journal of Physics B, vol. 34, no. 23, pp. 4877-4888, 2001.

[144] V. V. Serov, V. L. Derbov, B. B. Joulakian, and S. I. Vinitsky, "Wave-packet evolution approach to ionization of the hydrogen molecular ion by fast electrons," Physical Review A, vol. 63, no. 6, Article ID 062711, 8 pages, 2001.

[145] C. Champion, J. Hanssen, and P. A. Hervieux, "Theoretical differential and total cross sections of water-molecule ionization by electron impact," Physical Review A, vol. 65, no. 2, Article ID 022710, 9 pages, 2002.

[146] C. Champion, J. Hanssen, and P. A. Hervieux, "Electron impact ionization of water molecule," Journal of Chemical Physics, vol. 117, no. 1, pp. 197-204, 2002.

[147] M. J. Hussey and A. J. Murray, "Low energy (e, 2e) differential cross-section measurements on the $3 \sigma_{\mathrm{g}}$ and $1 \pi_{\mathrm{u}}$ molecular orbitals of $\mathrm{N}_{2}$," Journal of Physics B, vol. 35, no. 16, pp. 33993409, 2002.

[148] V. V. Serov, B. B. Joulakian, D. V. Pavlov, I. V. Puzynin, and S. I. Vinitsky, " $(\mathrm{e}, 2 \mathrm{e})$ ionization of $\mathrm{H}^{2+}$ by fast electron impact: application of the exact nonrelativistic two-center continuum wave," Physical Review A, vol. 65, no. 6, Article ID 62708, 7 pages, 2002.

[149] C. R. Stia, O. A. Fojón, P. F. Weck, J. Hanssen, B. Joulakian, and R. D. Rivarola, "Molecular three-continuum approximation for ionization of $\mathrm{H}_{2}$ by electron impact," Physical Review A, vol. 66, no. 5, Article ID 052709, 8 pages, 2002.

[150] P. F. Weck, O. A. Fojón, B. Joulakian, C. R. Stia, J. Hanssen, and R. D. Rivarola, "Two-center continuum approximation with correct boundary conditions for single-electron emission in $\mathrm{e}_{-}+\mathrm{H}^{2}$ collisions," Physical Review A, vol. 66, no. 1, Article ID 012711, 8 pages, 2002.

[151] O. A. Fojón, C. R. Stia, P. F. Weck, J. Hanssen, B. Joulakian, and R. D. Rivarola, "Ionization of molecular hydrogen by electron impact," Institute of Physics, vol. 172, pp. 11-20, 2003.

[152] S. Houamer, A. Mansouri, C. Dal Cappello, et al., "Second Born approximation for the ionization of $\mathrm{H}_{2}$ by electron impact," Journal of Physics B, vol. 36, no. 14, pp. 3009-3024, 2003.

[153] C. Champion, J. Hanssen, and P.-A. Hervieux, "Differential and total (e, 2e) cross sections of simple polyatomic molecules," Journal of Chemical Physics, vol. 121, no. 19, pp. 9423-9429, 2004. 
[154] O. Chuluunbaatar, B. B. Joulakian, K. Tsookhuu, and S. I. Vinitsky, "Modified two-centre continuum wavefunction: application to the dissociative ionization of $\mathrm{H}^{+}{ }_{2}$ by fast electrons," Journal of Physics B, vol. 37, no. 12, pp. 2607-2616, 2004.

[155] D. S. Milne-Brownlie, S. J. Cavanagh, B. Lohmann, C. Champion, P. A. Hervieux, and J. Hanssen, "Dynamics in electron-impact ionization of $\mathrm{H}_{2} \mathrm{O}$," Physical Review A, vol. 69, no. 3, Article ID 032701, 4 pages, 2004.

[156] J. Gao, D. H. Madison, and J. L. Peacher, "Fully differential cross sections for low-energy electron-impact ionization of nitrogen molecules," Physical Review A, vol. 72, no. 2, Article ID 020701, 4 pages, 2005.

[157] J. Gao, D. H. Madison, and J. L. Peacher, "Distorted wave born and three-body distorted wave born approximation calculations of the fully differential cross section for electronimpact ionization of nitrogen molecules," Journal of Chemical Physics, vol. 123, no. 20, Article ID 204314, 6 pages, 2005.

[158] J. Gao, D. H. Madison, and J. L. Peacher, "Interference effects for low-energy electron-impact ionization of nitrogen molecules," Physical Review A, vol. 72, no. 3, 5 pages, 2005.

[159] J. Gao, J. L. Peacher, and D. H. Madison, "An elementary method for calculating orientation-averaged fully differential electron-impact ionization cross sections for molecules," Journal of Chemical Physics, vol. 123, no. 20, Article ID 204302, 4 pages, 2005.

[160] M. J. Hussey and A. J. Murray, "Low energy (e, 2e) differential cross-section measurements on the $1 \pi_{\mathrm{u}}$ and $4 \sigma_{\mathrm{g}}$ molecular orbitals of $\mathrm{CO}_{2}$," Journal of Physics B, vol. 38, no. 16, pp. 2965-2977, 2005.

[161] A. J. Murray, "(e, 2e) studies of $\mathrm{H}_{2}$ in the intermediate energy regime," Journal of Physics B, vol. 38, no. 12, pp. 1999-2013, 2005.

[162] V. V. Serov, B. B. Joulakian, V. L. Derbov, and S. I. Vinitsky, "Ionization excitation of diatomic systems having two active electrons by fast electron impact: a probe to electron correlation," Journal of Physics B, vol. 38, no. 15, pp. 27652773, 2005.

[163] C. Dal Cappello, A. Mansouri, S. Houamer, and B. Joulakian, "Second-order effects in (e, 2e) ionization-excitation of $\mathrm{H}_{2}$," Journal of Physics B, vol. 39, no. 11, pp. 2431-2443, 2006.

[164] J. Gao, D. H. Madison, and J. L. Peacher, "Theoretical calculation of fully differential cross sections for electronimpact ionization of hydrogen molecules," Journal of Physics B, vol. 39, pp. 1275-1284, 2006.

[165] J. Gao, D. H. Madison, J. L. Peacher, A. J. Murray, and M. J. Hussey, "Experimental and theoretical (e, 2e) ionization cross sections for a hydrogen target at $75.3 \mathrm{eV}$ incident energy in a coplanar asymmetric geometry," Journal of Chemical Physics, vol. 124, no. 19, Article ID 194306, 2006.

[166] D. S. Milne-Brownlie, M. Foster, J. Gao, B. Lohmann, and D. H. Madison, "Young-type interference in $(e, 2 e)$ ionization of $\mathrm{H}_{2}$," Physical Review Letters, vol. 96, no. 23, Article ID 233201, 4 pages, 2006.

[167] A. J. Murray, M. J. Hussey, J. Gao, and D. H. Madison, “(e, 2e) ionization measurements from the $3 \sigma_{\mathrm{g}}$ and $2 \sigma$ states of $\mathrm{N}_{2}$ comparison between experiment and theoretical predictions of the effects of exchange, polarization and interference," Journal of Physics B, vol. 39, no. 18, pp. 3945-3956, 2006.

[168] C. Kaiser, D. Spieker, J. Gao, M. Hussey, A. Murray, and D. H. Madison, "Coplanar symmetric and asymmetric electron impact ionization studies from the $1 b_{1}$ state of $\mathrm{H}_{2} \mathrm{O}$ at low to intermediate impact energies," Journal of Physics B, vol. 40, no. 13, pp. 2563-2576, 2007.
[169] A. J. Murray, M. J. Hussey, C. Kaiser, J. Gao, J. L. Peacher, and D. H. Madison, "Electron impact ionization of molecules at low to intermediate energies-a search for Young's double slit type interferences," Journal of Electron Spectroscopy and Related Phenomena, vol. 161, no. 1-3, pp. 11-16, 2007.

[170] A. Naja, E. M. Staicu-Casagrande, A. Lahmam-Bennani, et al., "Triply differential (e, 2e) cross sections for ionization of the nitrogen molecule at large energy transfer," Journal of Physics B, vol. 40, no. 18, pp. 3775-3783, 2007.

[171] V. V. Serov, V. L. Derbov, B. B. Joulakian, and S. I. Vinitsky, "Wave-packet-evolution approach for single and double ionization of two-electron systems by fast electrons," Physical Review A, vol. 75, no. 1, Article ID 012715, 9 pages, 2007.

[172] E. M. Staicu-Casagrande, A. Naja, X. G. Ren, et al., "Coincidence angular correlation in electron impact single or double ionisation of atoms and molecules," Journal of Physics, vol. 88, no. 1, Article ID 012010, 7 pages, 2007.

[173] C. Champion, O. Boudrioua, and D. Dal Cappello, "Water molecule ionization by charged particles: a short review," Journal of Physics, vol. 101, Article ID 012010, 10 pages, 2008.

[174] O. Chuluunbaatar, B. B. Joulakian, I. V. Puzynin, Kh. Tsookhuu, and S. I. Vinitsky, "Modified two-centre continuum wavefunction: application to the dissociative double ionization of $\mathrm{H}_{2}$ by electron impact," Journal of Physics B, vol. 41, no. 1, Article ID 015204, 6 pages, 2008.

[175] E. M. Staicu-Casagrande, A. Naja, F. Mezdari, et al., “(e, 2e) ionization of helium and the hydrogen molecule: signature of two-centre interference effects," Journal of Physics B, vol. 41, no. 2, Article ID 025204, 7 pages, 2008.

[176] E. M. Staicu-Casagrande, A. Naja, A. Lahmam-Bennani, A. S. Kheifets, D. H. Madison, and B. Joulakian, "New (e, 2e) studies of atomic and molecular targets," Journal of Physics, vol. 141, Article ID 012016, 7 pages, 2008.

[177] J. Colgan, M. S. Pindzola, F. Robicheaux, C. Kaiser, A. J. Murray, and D. H. Madison, "Differential cross sections for the ionization of oriented $\mathrm{H}_{2}$ molecules by electron impact," Physical Review Letters, vol. 101, no. 23, Article ID 233201, 4 pages, 2008.

[178] J. Colgan, O. Al-Hagan, D. H. Madison, C. Kaiser, A. J. Murray, and M. S. Pindzola, "Triple differential cross sections for the electron-impact ionization of $\mathrm{H}_{2}$ molecules for equal and unequal outgoing electron energies," Physical Review A, vol. 79, no. 5, Article ID 052704, 7 pages, 2009.

[179] J. Colgan, O. Al-Hagan, D. H. Madison, A. J. Murray, and M. S. Pindzola, "Deep interference minima in noncoplanar triple differential cross sections for the electronimpact ionization of small atoms and molecules," Journal of Physics B, vol. 42, no. 17, Article ID 171001, 6 pages, 2009.

[180] I. Kada, A. Mansouri, C. Dal Cappello, P. A. Hervieux, and A. C. Roy, "Ab initio calculation of differential cross sections for the double ionization of water vapour by electron impact," Journal of Physics B, vol. 42, no. 2, Article ID 025201, 9 pages, 2009.

[181] A. Mansouri, C. Dal Cappello, I. Kada, C. Champion, and A. C. Roy, "(e, 3e) and (e,3-1e) differential cross sections for the double ionization of water molecule," Physics Letters A, vol. 373, no. 35, pp. 3151-3157, 2009.

[182] I. E. McCarthy and E. Weigold, “(e, 2e) spectroscopy," Physics Reports, vol. 27, no. 6, pp. 275-371, 1976.

[183] I. E. McCarthy and E. Weigold, "Wavefunction mapping in collision experiments," Reports on Progress in Physics, vol. 51, no. 3, pp. 299-392, 1988. 
[184] I. E. McCarthy and A. M. Rossi, "Momentum-space calculation of electron-molecule scattering," Physical Review A, vol. 49, no. 6, pp. 4645-4652, 1994.

[185] F. Robicheaux, "Electron impact ionization of $\mathrm{H}_{2}^{+}$," Journal of Physics B, vol. 29, no. 4, pp. 779-790, 1996.

[186] H. Deutsch, K. Becker, R. Basner, M. Schmidt, and T. D. Märk, "Application of the modified additivity rule to the calculation of electron-impact ionization cross sections of complex molecules," Journal of Physical Chemistry A, vol. 102, no. 45, pp. 8819-8826, 1998.

[187] S. Rioual, G. Nguyen Vien, and A. Pochat, "Ionization in coplanar symmetric (e, 2e) experiments of $\mathrm{N}_{2}$ and $\mathrm{CO}$ at intermediate energies," Physical Review A, vol. 54, no. 6, pp. 4968-4977, 1996.

[188] A. L. Monzani, L. E. Machado, M.-T. Lee, and A. M. Machado, "Intermediate-energy electron-impact ionization of molecules," Physical Review A, vol. 60, no. 1, pp. R21-R24, 1999.

[189] M. Chérid, A. Lahmam-Bennani, A. Duguet, et al., "Triple differential cross sections for molecular hydrogen, both under Bethe ridge conditions and in the dipolar regime. Experiments and theory," Journal of Physics B, vol. 22, no. 21, pp. 3483-3499, 1989.

[190] J. B. Furness and I. E. McCarthy, "Semiphenomenological optical model for electron scattering on atoms," Journal of Physics B, vol. 6, no. 11, pp. 2280-2291, 1973.

[191] M. E. Riley and D. G. Truhlar, "Approximations for the exchange potential in electron scattering," The Journal of Chemical Physics, vol. 63, no. 5, pp. 2182-2191, 1975.

[192] J. P. Perdew and A. Zunger, "Self-interaction correction to density-functional approximations for many-electron systems," Physical Review B, vol. 23, no. 10, pp. 5048-5079, 1981.

[193] N. T. Padial and D. W. Norcross, "Parameter-free model of the correlation-polarization potential for electron-molecule collisions," Physical Review A, vol. 29, no. 4, pp. 1742-1748, 1984.

[194] J. Rasch, C. T. Whelan, R. J. Allan, and H. R. J. Watlers, "An explanation of the structure observed in out-of-plane symmetric measurements on Helium," in Coincidence Studies of Electron and Photon Impact ionization, Whelan and Walters, Eds., p. 185193, Plenum Press, New York, NY, USA, 1997.

[195] L. R. Hargreaves, C. Colyer, M. A. Stevenson, et al., “(e, 2e) study of two-center interference effects in the ionization of $\mathrm{N}_{2}$," Physical Review A, vol. 80, no. 6, Article ID 062704, 7 pages, 2009.

[196] K. L. Nixon, A. J. Murray, O. Al-Hagan, D. H. Madison, and C. Ning, "Low-energy symmetric coplanar and symmetric non-coplanar (e, 2e) studies from the $3 \mathrm{a}_{1}$ state of $\mathrm{H}_{2} \mathrm{O}$," Journal of Physics B, vol. 43, no. 3, Article ID 035201, 7 pages, 2010.

[197] C. J. Colyer, M. A. Stevenson, O. Al-Hagan, D. H. Madison, C. G. Ning, and B. Lohmann, "Dynamical (e, 2e) studies of formic acid," Journal of Physics B, vol. 42, no. 23, Article ID 235207, 6 pages, 2009.

[198] K. L. Nixon, W. D. Lawrance, and M. J. Brunger, "Electron momentum spectroscopy of formic acid," Chemical Physics Letters, vol. 474, no. 1-3, pp. 23-27, 2009. 

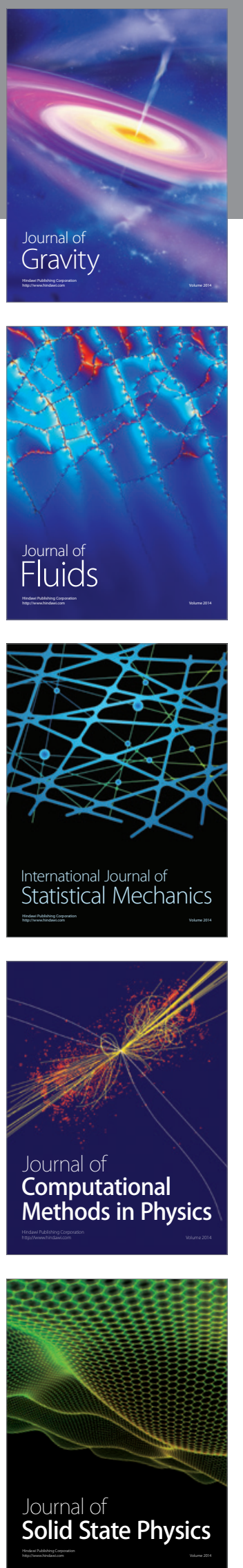

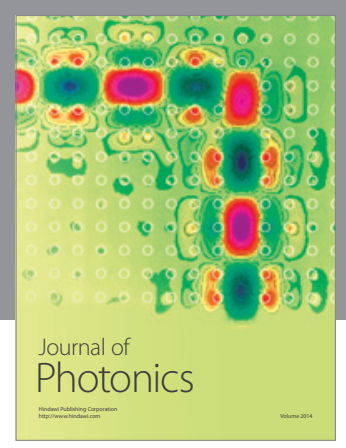

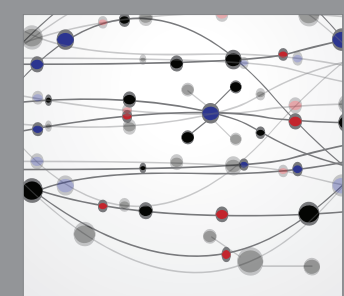

The Scientific World Journal
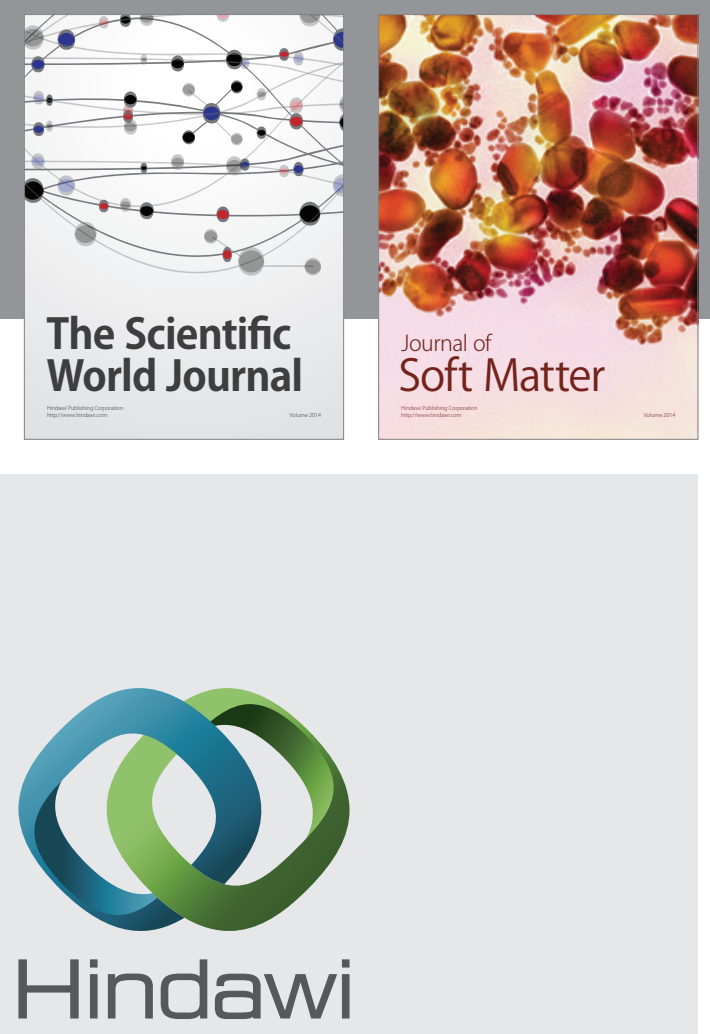

Submit your manuscripts at

http://www.hindawi.com
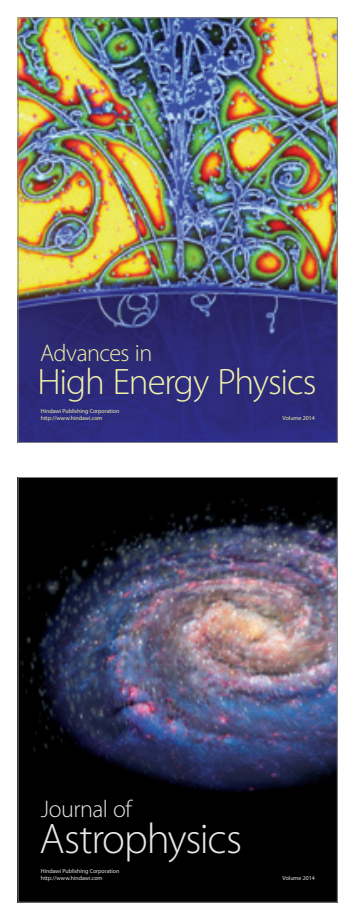
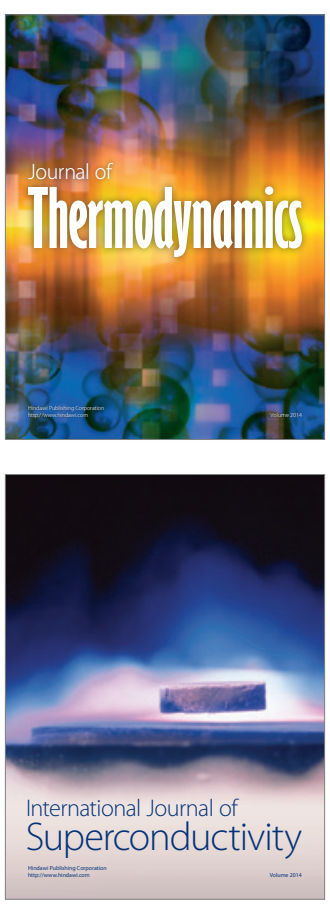
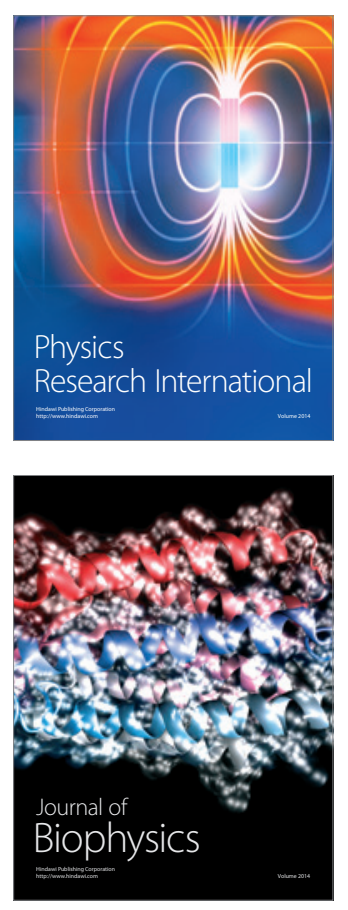
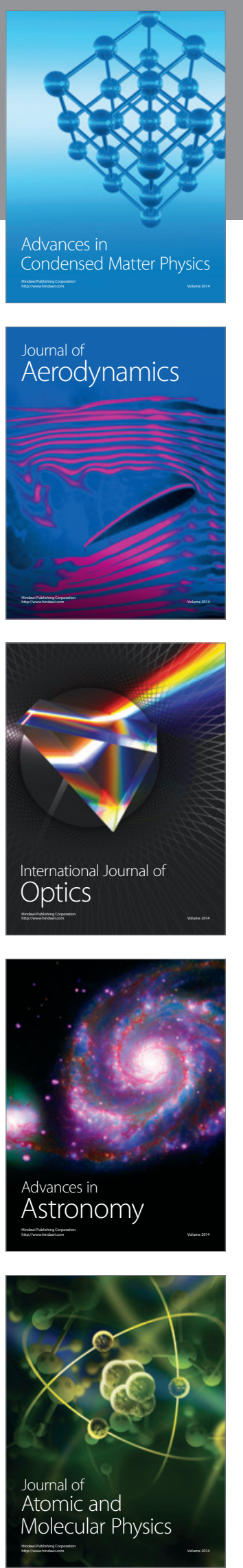